\title{
Hall Current and Joule Heating Effects on Peristaltic Flow of a Sisko Fluid with Mild Stenosis through a Porous Medium in a Tapered Artery with Slip and Convective Boundary Conditions
}

(Arus Hall dan Kesan Pemanasan Joule pada Aliran Peristalsis Bendalir Sisko dengan Stenosis Lembap melalui Medium Berliang dalam Arteri Tirus dengan Syarat Gelinciran dan Syarat Sempadan Olakan)

\author{
NABIL T.M. EL-DABE \& DOAA R. MOSTAPHA*
}

\begin{abstract}
This work is arranged to investigate the Hall current and Joule heating effects on peristaltic flow of a Sisko fluid through a porous medium. The streaming is through tapered artery with mild stenosis. The influences of radiative heat transfer and chemical reactions are taken in consideration. Convective conditions are considered for heat and mass transfer. However, the slip conditions are adopted for the velocity distribution. The combined effects of viscous dissipation and radiation in energy expression are presented. Soret and Dufour features produce the coupled differential systems. The presumptions of the long wavelength and low Reynolds number are adopted to examine the governing equations of motion. The analytical solutions of these equations are given by two methods. The first one uses regular perturbation technique, which based on small wave number for small artery. The second one is based on utilizing the Homotopy perturbation technique. The approximate analytical solutions of the pressure rise and friction force are predestined along a numerical integration. The influences of various physical parameters of the problem are debated and depicted graphically through a set of figures. It is found that the axial velocity increases with the increase of Hall current parameter and with the decrease of Hartmann number. Also, it can be observed that the Brickmann number and Dufour number give rise to the fluid temperature. Meanwhile, reverse effect is observed towards concentration for both Schmidt number and chemical reaction number. Furthermore, the stream lines are graphically shown.
\end{abstract}

Keywords: Hall current; Joule heating; peristaltic flow; porous medium; Sisko model

\section{ABSTRAK}

Kajian ini mengkaji arus Hall dan kesan pemanasan Joule terhadap aliran peristalsis bendalir Sisko melalui medium berliang. Aliran adalah melalui arteri tirus dengan stenosis lembut. Pengaruh pemindahan haba sinaran dan tindak balas kimia dipertimbangkan. Syarat perolakan dipertimbangkan bagi pemindahan haba dan jisim. Walau bagaimanapun, syarat gelinciran digunakan dalam taburan halaju. Kesan gabungan lesapan likat dan radiasi dalam ungkapan tenaga dipersembahkan. Ciri-ciri Soret dan Dufour menghasilkan sistem persamaan pembezaan terganding. Andaian panjang gelombang dan nombor Reynolds yang rendah digunakan untuk mengkaji persamaan yang mengawal gerakan. Penyelesaian beranalisis persamaan ini diberikan oleh dua kaedah. Yang pertama menggunakan teknik usikan biasa, yang berdasarkan nombor gelombang kecil untuk arteri kecil. Yang kedua adalah berdasarkan teknik usikan homotopi. Penyelesaian analisis anggaran kenaikan tekanan dan daya geseran ditentukan menggunakan kamiran berangka. Pengaruh pelbagai parameter fizikal bagi masalah ini dibahas dan digambarkan secara grafik. Didapati bahawa halaju bertambah dengan peningkatan parameter arus Hall dan dengan penurunan nombor Hartmann. Juga, boleh diperhatikan bahawa nombor Brickmann dan nombor Dufour meningkatkan suhu bendalir. Sementara itu, kesan bertentangan diperhatikan terhadap kepekatan untuk kedua-dua nombor Schmidt dan nombor tindak balas kimia. Juga, garis arus ditunjukkan secara grafik.

Kata kunci: Aliran peristalsis; arus Hall; pemanasan Joule; medium berliang; model Sisko

\section{INTRODUCTION}

The non-Newtonian fluids have a great significance in industrial and technological applications. Sisko (1958) proposed a new model in investigating the nonNewtonian fluid, which is later known as a Sisko fluid.
It is a model which amalgamates the features of viscous and generalization of power law models. Sisko model is capable of describing shear thinning and thickening phenomena. Sisko fluid is an example of viscoelastic materials that include waterborne coatings, metallic 
automotive, cement slurries, lubricating greases, psueodoplastic fluids, and drilling fluids (Khan et al. 2010). It can determine many typical characteristics of Newtonian and non-Newtonian fluids by choosing different material parameters. Thus, it can be considered as a model of blood.

The mechanism of non-Newtonian fluids flow in porous medium has acquired a major attention in previous and current research. The range of applications involves extraction of crude oils, well drilling, underground oil displacement, aquifer contamination, and soil re-mediation (Longo \& Di Federico 2015). Furthermore, the flow through porous medium includes many applications, such as material science, petroleum industry, chemical engineering, bile duct, the human lung, gall bladder with stones, small blood vessels and soil mechanics (Lauriola et al. 2018). Profound studies in the physics of porous medium were scrutinized by Di Federicoa et al. (2017), Eldabe et al. (2007) and Scheidegger (1963).

Peristaltic transport is a kind of material transmission given by a progressive wave of area contraction and expansion along the wall of the tubes. Peristalsis has a requisite function in transporting many biological fluids (Burns \& Parkes 1967), such as urine transmission from kidney to bladder, food transmission through oesophagus, blood transmission in heart and bile transmission in the bile duct. Several theoretical and experimental articles inspected the peristaltic flows, such as Fung and Yih (1969), Hayat et al. (2014), Manton (1975) and Shapiro et al. (1969). The previously mentioned works focused on several assumptions, such as long wavelength approximation, low Reynolds number and small amplitude ratio.

In light of the aforementioned studies, the analysis of blood flow through stenosed arteries is very fundamental. Stenosis represents narrowing of any body passage (Ang \& Mazumdar 1997). This narrowing results in several diseases, such as cerebral strokes, myocardial infarction, and heart failure by reducing or occluding the blood pressure (Nichols \& Orourke 1973). Moreover, stenosed artery shows higher level of stresses and resistance than those in the normal ones. The description of the mechanism of the blood flow characteristics through stenosed arteries has attracted the attention of many scientists such as Chakravarty et al. (1995), Jamil (2018) and Verma and Parihar (2010).

Heat transfer has a vital role in investigating chemical engineering and peristaltic transport. Mobile phones, radioactive treatments and radio-frequency therapy are applications of heat transfer (Arora 1997). The influence of heat transfers on a peristaltic transport of Herschel-Bulkley fluid in a non-uniform inclined tube was examined by Nadeem and Akbar (2009). Recently, the effects of heat and mass transfer on bio-fluids were examined both theoretically and practically, by Chakravarty and Sen (2005), Eldabe et al. (2008) and Ijaz et al. (2008).

The scrutinize of heat and mass transfer with chemical reactions is crucial in the chemical and hydrometallurgical industries. Additionally, the influences of thermal radiation on forced and free convection flow are fundamental in the content of space technology and high temperature processes. Through the Newtonian fluids, the effects of thermaldiffusion (Soret) and diffusion-thermo (Dufour) might be ignored. In contrast, for the non-Newtonian fluids, it is inconvenient to exclude these effects as shown by Hayat et al. (2019, 2018a, 2016) and Pal and Talukdar (2011). Moreover, study of heat and mass transfer with convective boundary condition is very significant due to its essential applications. These applications are hemodialysis and oxygenation, hypothermia treatment, blood pump in heart-lung machines, transport of corrosive fluids, and the destruction of undesirable tissues (Sadaf \& Nadeem 2017; Sohail et al. 2019).

In recent years, the investigation of magnetohydrodynamic (MHD) flow problems has acquired considerable interest because of its comprehensive applications in the fields of engineering and medicine. MHD possesses has many applications, such as cell separation, magnetic wound, cancer tumor treatment, and development of magnetic tracers (Abdelsalam \& Bhatti 2019). Several theoretical articles have examined the effects of magnetic field on peristaltic flow such as Bhatti et al. (2016), Eldabe et al. (2015, 2014, 2013), Hayat et al. (2018b), Mehmood et al. (2014), and Rashid et al. (2019). Hall current is another important branch of MHD peristaltic flow. This effect appears when the magnetic field is high. It has numerous applications in engineering field; such as power generators, heating elements, Hall accelerators MHD generators and in flight MHD (Abdelsalam \& Bhatti 2018). Moreover, Joule heating happens when the energy of an electric current is changed into heat through a resistance. It has practical applications in biomedical and nuclear engineering, fluid industries, and geophysical flows (Hayat et al. 2017, 2016b).

In recent years, body of research (Hayat et al. 2016a; Pal \& Talukdar 2011) focused on studying the effects of chemical reaction, thermal radiation, Soret and Dufour effects on a viscous dissipating fluid. Furthermore, Hayat et al. (2017) and Sadaf and Nadeem (2017) examined the influences of convective boundary conditions and the influences of Hall current and Joule heating on peristaltic transport. In addition, there is a growing interest in investigating Sisko fluid because of its significance in studying the behavior of blood. El- 
dabe et al. (2016) studied the influence of partial slip on peristaltic flow of a Sisko fluid through a porous medium. They studied the flow in a tapered artery having a mild stenosis. Therefore, the aim of the present study is to investigate the influences of Hall current and Joule heating on peristaltic flow of a Sisko fluid through a porous medium. The streaming of flow is through a tapered artery having mild stenosis. Furthermore, slip condition for velocity distribution and convective conditions for heat and mass transfer are also taken into account. In addition, the effects of radiation, viscous dissipation and chemical reactions are also considered. Additionally, Soret and Dufour effects are discussed. The governing equations of motion are simplified by utilizing the long wavelength and low Reynolds number assumptions. These equations are analytically solved in accordance with the appropriate boundary conditions by using two methods: the first one is a perturbation technique, and the second method is Homtopy perturbation technique. The distributions of the axial velocity, the stream function, temperature and concentration are obtained. The pressure rise and friction force are predestined by using numerical integration. Numerical calculations are analyzed to obtain the effects of several parameters of the problem at hand. It is found out that the axial velocity decreases with the increase of Hartmann number. This shows that if we increase the strength of magnetic field, the flow of blood will be decreased. Additionally, it is observed that the axial velocity increases with the increase of Hall current parameter. Moreover, it is found out that both Soret and Dufour numbers give rise to the fluid temperature towards concentration and vice versa. Moreover, it is depicted that the increase in mean flow rate decreases the pressure rise. Therefore, the relation between pressure rise and mean flow rate is linear.

\section{MATERIALS AND METHODS}

Consider an unsteady motion of a Sisko fluid flowing through a tapered tube. This tube has a sinusoidal wave traveling down its wall with mild stenosis. The flow is streaming through a porous medium. Fluid is acted upon by strong magnetic field of uniform strength $B_{0}$, (Figure 1). However, electric field effect is not applied. Meanwhile, the Hall and Joule heating effects are considered. Radiative heat and mass transfer are imposed with the effects of viscous dissipation. Cylindrical polar co-ordinates system $(R, \theta, Z)$ is employed, so that the $Z$-axis coincides with the axis of the tubes. Presence of Hall current produces a force in $\theta$-direction. Therefore, the flow becomes three dimensions. However, there are no effects on flow, heat transfer, and concentration properties in $\theta$ direction. The tube is convectively heated. Also, the convective mass condition is applied. Further, it is presumed that the wall of tube is heated by a temperature $T^{*}$ and concentration $C^{*}$. This presumption generates heat and mass transfer coefficients $h_{t}$ and $h_{c}$. Chemical reaction, Soret and Dufour effects are also taken into account. The magnetic Reynolds number is elected to be very small. Therefore, the induced magnetic field is smaller in comparison to the applied magnetic field. Thus, the induced magnetic field is not emulated. The slip condition is also considered for velocity distribution.

As stated in Verma and Parihar (2010), the effective radius of the tapered artery $R(z)$, is taken as follows:

$R(\mathrm{z})=\left\{\begin{array}{lc}R_{1}-\delta m_{1}(Z+L+d) & -\mathrm{L} \leq \mathrm{Z}<-\mathrm{Z}_{0}, \\ R_{1}-\delta m_{1}(Z+L+d)-\frac{H_{0}}{2}\left[1+\cos \frac{\pi \mathrm{Z}}{Z_{0}}\right] & -\mathrm{Z}_{0} \leq \mathrm{Z} \leq \mathrm{Z}_{0}, \\ R_{1}-\delta m_{1}(Z+L+d) & \mathrm{Z}_{0}<\mathrm{Z} \leq \mathrm{d},\end{array}\right.$

where $R_{1}$ is the radius of the artery; $\delta=\frac{R_{1}}{\lambda}$, is the wave length; $H_{0}=h \cos \phi$ is the hight of the stenosis in the tapered artery; $\phi$ is the angle of tapering; $h$ is the maximum height of the stenosis; $z_{0}$ is the half-length of the stenosis; and $m_{1}=\tan \phi$ is the slope of the tapered artery.

The ratio among the height of the stenosis and the radius of the normal artery is much less than unity $\left(\frac{H}{-}\right)$. The artery is assumed to be of finite length $L+$ $d$ (Chakravarty \& Sen 2005). This paper focus on all possibilities of different shapes of the artery viz, the converging tapering $\phi<0$, non-tapered artery $\phi=0$ and the diverging tapering $\phi>0$ (Mandal 2005).

As stated in Sisko (1958), the deemed nonNewtonian fluid is introduced by Sisko model. Therefore, the constitutive equation can be written in the following form:

$$
\underline{S}=\left(a_{1}+b_{1}(\sqrt{\Theta})^{n-1}\right) \underline{A},
$$

where $\underline{S}$ is the stress deviator; $\Theta=\frac{1}{2} \operatorname{tr}(\underline{A})^{2} ; \operatorname{tr}(\underline{A})^{2}$ is defined as the sum of elements in main diagonal of $(\underline{A})^{2} ; n$ is the power index; and $a_{1}$ and $b_{1}$ are the material constant for Sisko fluid,

$$
\underline{A}=L+L^{T}
$$

is the rate of strain tensor, $\underline{V}=(U, 0, W)$ is the velocity field and $\mathrm{L}=\nabla \underline{V}$. 


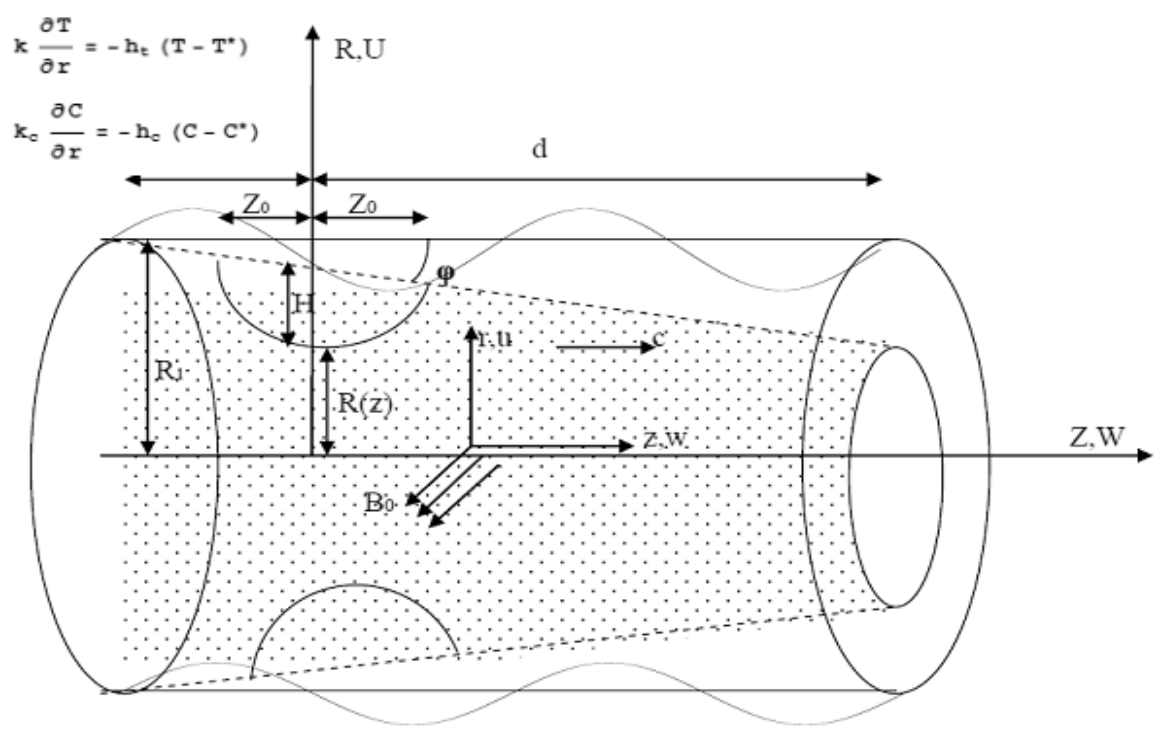

FIGURE 1. Sketch of the physical situation of the problem

Since the density of the fluid $\rho$ is presumed to be uniform, the incompressibility condition can be written as

$$
\nabla \cdot \underline{V}=0 .
$$

The equation of motion can be written as

$$
\rho\left(\frac{\partial \underline{V}}{\partial t}+(\underline{V} \cdot \nabla) \underline{V}\right)=-\nabla P+\nabla \cdot \underline{S}+\underline{J} \wedge \underline{B}-\frac{a_{1}}{k_{1}} \underline{V} .
$$

where $P$ is the pressure; $J \wedge \underline{B}$ is Lorentz force; $J$ is current density; $\underline{B}=\left(0, B_{0}, 0\right)$ is applied magnetic field; and $k_{1}$ is the permeability parameter of porous media.

By assuming the Hall effects, the current density (Abdelsalam \& Bhatti 2018) can be introduced under the assumption of no polarization voltage (i.e. the total electric field $\underline{E}=0$ ) as follows,

$$
\underline{J}=\sigma\left(\underline{V} \wedge \underline{B}-\frac{1}{e n_{c}} J \wedge \underline{B}\right) .
$$

where $\sigma$ is the electric conductivity; $e$ can be defined as the electron charge; $n$ represents the number density of free electrons and $\frac{1}{e n_{c}}$ is called the Hall factor. The equation of energy with Joule heating, thermal radiation and Dufour effects (Arora 1997; Hayat et al. 2016a; Sohail et al. 2019) is represented as follows:

$$
\rho c_{p}\left(\frac{\partial T}{\partial t}+\underline{V} \cdot \nabla T\right)=\Phi_{1}+K \nabla^{2} T-\nabla \cdot q_{r}+\frac{1}{\sigma} J \cdot \underline{J}+\frac{D K_{T}}{C_{s}} \nabla^{2} C+\frac{a_{1}}{k_{1}}(U+W)|\underline{V}|,
$$

where $T$ is the temperature; $k=\frac{K}{\rho c_{p}}$ is the thermometric conductivity; $K$ is the thermal conductivity; $c_{p}$ is the specific heat; $\Phi_{1}=S_{i j} \frac{\partial V_{i}}{\partial X_{j}}$ is the dissipation factor; $K_{T}$ is thermal diffusion ratio; $C_{S}$ is the concentration susceptibility; $C$ is the concentration distribution; $|\underline{V}|=$ $\sqrt{U^{2}+W^{2}}$; and $q_{r}$ is radiative heat flux.
Utilizing the Rosseland diffusion flux model (Rohsenow 1998), the integro-differential equation for radiative transfer may be turned to a Fourier-type diffusion equation. It is essential to observe that the Rosseland model is quite delicate in case of thermal radiation (scattering or absorption). The refractive index of the fluid-particle suspension is presumed to be constant. Therefore, the nonlinear radiative heat flux may be represented effectively as:

$$
q_{r}=-\frac{4 \sigma_{0}}{3 K_{0}} \frac{\partial T^{4}}{\partial r} .
$$

where $K_{0}$ is the Rosseland absorption coefficient and $\sigma_{0}$ is Stefan Boltzman constant.

The temperature differences are presumed to be adequately small. Therefore, $\left(T^{4}\right)$ can be expressed as a linear function of temperature. By using Talyor series for $T^{4}$ about the mean fluid temperature $T_{m}$ and ignoring the higher order terms, the nonlinear radiative heat flux is reduced to following linear form

$$
q_{r}=-\frac{16 \sigma_{0}}{3 K_{0}} T_{m} \frac{\partial T}{\partial r}
$$

The equation of concentration with chemical reaction and Soret effects (Hayat et al. 2016a) is represented as follows:

$$
\frac{\partial C}{\partial t}+\underline{V} \cdot \nabla C=D \nabla^{2} C+\frac{D K_{T}}{T_{m}} \nabla^{2} T-L_{c}\left(C-C^{*}\right),
$$

where $D$ is the coefficient of thermal diffusivity; $K_{\mathrm{T}}$ is the thermal diffusion; $T_{m}$ is the mean fluid temperature; and $L_{c}$ is reaction rate constant.

The geometry of the peristaltic wall surface may be defined as (Fung \& Yin 1969) 


$$
H_{1}=a \cos \frac{2 \pi}{\lambda}\left(Z-\frac{k t}{R_{1}}\right)
$$

where $\alpha$ is the wave amplitude; $\lambda$ is the wave length; and $\frac{k t}{R_{1}}$ is the wave speed.

The Convective boundary conditions with partial slip for the considered flow analysis (El-dabe et al. 2016; Hayat et al. 2016b; Sohail et al. 2019) can be listed as:

$$
\begin{gathered}
U=\frac{\partial H_{1}}{\partial t}, W=-\gamma S_{R Z}, K \frac{\partial T}{\partial r}=-h_{t}\left(T-T_{*}\right), D \frac{\partial C}{\partial r}=-h_{c}\left(C-C_{*}\right) \\
R_{2}=R(z)+H_{1}, \\
U=0, \frac{\partial W}{\partial R}=0, \frac{\partial T}{\partial R}=0, \frac{\partial C}{\partial R}=0 \text { at } R=0,
\end{gathered}
$$

where $\gamma$ is the slip parameter; $h_{t}$ is called heat transfer coefficient and it represents the proportionality constant among the heat flux and the thermodynamic driving force for the heat flow. Meanwhile, $h_{c}$ is defined as mass transfer coefficient and it is utilized to depict the ratio between the actual mass flux of a species into or out of a flowing fluid and the driving force for this flux.

The instantaneous volume flow rate, in the fixed coordinate system, is defined as:

$$
Q=2 \pi \int_{0}^{R_{2}} W R d R,
$$

where $R_{2}$ is a function of $\mathrm{Z}$ and $\mathrm{t}$.

The time averaged $\hat{Q}$ (time mean flow) over period $\tau=\frac{\lambda R_{1}}{k}$ at a fixed Z-position is defined as

$$
\widehat{Q}=\frac{1}{\tau} \int_{0}^{\tau} Q d \tau
$$

We presume that the tube length is an integral multiple of wavelength $\lambda$. In addition, the pressure difference through the ends of the tube is taken to be a constant. Thus, the unsteady flow in laboratory frame $(R, 0, Z)$ turns to steady flow in the wave frame $(r, 0, z)$. The transformation between these two frames can be written as

$$
u=U, w=W-\frac{k}{R_{1}}, \quad t r=R \text { and } z=Z-\frac{k}{R_{1}} t .
$$

It is adequate to reformulate the previous equations in an appropriate dimensionless form. This can be done in a number of methods relying primarily on the choice of the characteristic length, time, and mass. Consider the following dimensionless forms relying on the characteristic length $R_{1}, \lambda$ and the characteristic mass $M$. The other dimensionless quantities are displayed as

$$
\begin{aligned}
& \begin{array}{l}
\bar{r}=\frac{r}{R_{1}}, \bar{z}=\frac{z}{\lambda}, \bar{u}=\frac{u R_{1}}{k \delta}, \bar{w}=\frac{w R_{1}}{k}, \overline{H_{1}}=\frac{H_{1}}{R_{1}}, \delta=\frac{R_{1}}{\lambda}, \bar{S}=\frac{S R_{1}^{2}}{a_{1} k}, \overline{z_{0}} \\
\quad=\frac{z_{0}}{\lambda}, \bar{L}=\frac{L}{\lambda}, \bar{h}=\frac{h}{R_{1}^{\prime}}
\end{array} \\
& \bar{d}=\frac{d}{\lambda}, \overline{R(z)}=\frac{R(z)}{R_{1}}, \bar{P}=\frac{P R_{1}^{3}}{a_{1} \lambda k}, \bar{T}=\frac{T-T^{*}}{\beta R_{1}}, \bar{C}=\frac{C-C^{*}}{C^{*}}, \overline{k_{1}}=\frac{k_{1}}{R_{1}^{2}} \\
& \text { and } \bar{Q}=\frac{Q}{2 \pi R_{1} k} .
\end{aligned}
$$

Consider another dimensionless parameters: $b_{1}^{*}=\frac{b_{1}}{a_{1}}\left(\frac{k}{R_{1}^{2}}\right)^{n-1}$ is the Sisko parameter, $R_{e}=\frac{k}{v}$ is the Reynold's number, $v=\frac{a_{1}}{\rho}$ is the kinematic viscosity, $a_{0}$ $=\mathrm{a} / R_{1}$ is the amplitude ratio, $H^{2}=\frac{\sigma B_{0}^{2} R_{1}^{2}}{a_{1}}$ is the Hartmann number, $N^{2}=\frac{H^{2}}{1+m^{2}}+\frac{1}{k_{1}}, m=\frac{\sigma B_{0}}{e n_{c}}$ is the Hall current parameter, $P_{r}=\frac{a_{1}}{\rho k}$ is the Prandtl number, $\beta$ is the adverse temperature gradient, $E_{c}=\frac{k^{2}}{R_{1}^{3} c_{p} \beta}$ is the Eckert number, $B_{r}$ $=P_{r} E_{c}$ is the Brickmann number, $R_{n}=1+\frac{16 \sigma_{0} T^{* 3}}{3 K K_{0}}$ is the radiation parameter, $D_{u}=\frac{D K_{T} C^{*}}{a_{1} C_{s} \beta R_{1} c_{p}}$ is the Dufour number, $S_{c}=\frac{k}{D}$ is the Schmidt number, $S_{r}=\frac{D K_{T} \beta R_{1}}{k T_{m} C^{*}}$ is the Soret number, $\alpha=\frac{L_{c} R_{1}^{2}}{k}$ is the chemical reaction number, $\gamma^{*}=\frac{\gamma a_{1}}{R_{1}}$ is the non-dimensional slip parameters, $\beta_{t}=\frac{h_{t} R_{1}}{K}$ is the heat transfer Biot number and $\beta_{c}=\frac{h_{c} R_{1}}{D}$ is the mass transfer Biot number. The bars mark indicates to the dimensionless quantities. From now on, these will be canceled for simplicity.

According to the presumption of Verma and Parihar (2010), we shall suppose that the radial velocity $u$ is very small in rapprochement with the axial one $w$. As well, the variation in the $z$-direction is less than that in the radial one. Thus, we may postulate that $u<<w$ and $\frac{\partial w}{\partial z}<<\frac{\partial w}{\partial r}$. Moreover, the hypothesis of long wave length approximation $\delta<<1$ is also presumed. Now, $\delta$ is very very small so that it can tend to zero. Therefore, the dimensionless governing (5), (7), (10) and (14) with the appropriate boundary conditions (12) and (13), by using this assumption, may be expressed as: $r$-component of the velocity:

$$
\frac{\partial P}{\partial r}=0
$$

equation (17) indicates that $P$ is a function of $z$ only. $z$-component:

$$
\frac{d P}{d z}=\frac{1}{r} \frac{\partial}{\partial r}\left(r\left(\frac{\partial w}{\partial r}+b_{1}^{*}\left(\frac{\partial w}{\partial r}\right)^{n}\right)\right)-N^{2} w
$$

heat equation:

$$
\begin{aligned}
\frac{1}{r} \frac{\partial}{\partial r}\left(r \frac{\partial T}{\partial r}\right) & +\frac{D_{u} P_{r}}{1+R_{n}} \frac{1}{r} \frac{\partial}{\partial r}\left(r \frac{\partial C}{\partial r}\right)+\frac{B_{r}}{1+R_{n}}\left(\left(\frac{\partial w}{\partial r}\right)^{2}+b_{1}^{*}\left(\frac{\partial w}{\partial r}\right)^{n+1}\right) \\
& +\frac{B_{r} N^{2}}{1+R_{n}} w^{2}=0
\end{aligned}
$$

concentration equation:

$$
\frac{1}{r} \frac{\partial}{\partial r}\left(r \frac{\partial C}{\partial r}\right)+\frac{S_{r} S_{c}}{r} \frac{\partial}{\partial r}\left(r \frac{\partial T}{\partial r}\right)-\alpha S_{c} C=0,
$$

and the dimensionless volume flow rate in the moving coordinate system become:

$$
q=\int_{0}^{r_{2}} w r d r
$$

The dimensionless boundary conditions are then become

$$
\begin{gathered}
u=2 \pi \varepsilon \sin 2 \pi z, w=-1-\gamma^{*}\left(\frac{\partial w}{\partial r}+b_{1}^{*}\left(\frac{\partial w}{\partial r}\right)^{n}\right), \frac{\partial T}{\partial r}=-\beta_{t} T, \\
\frac{\partial C}{\partial r}=-\beta_{c} C \quad r_{2}=r(z)+H_{1},
\end{gathered}
$$




$$
u=0, \frac{\partial w}{\partial r}=0, \frac{\partial T}{\partial r}=0, \frac{\partial C}{\partial r}=0 \text { at } r=0,
$$

where

$$
H_{1}=\varepsilon \cos 2 \pi z
$$

The dimensionless effective radius of the tube $R(z)$ becomes:

$$
R(z)=\left\{\begin{array}{lc}
1-m_{1}(z+L+d) & -\mathrm{L} \leq \mathrm{z}<-\mathrm{z}_{0} \\
1-m_{1}(z+L+d)-\frac{h \cos \phi}{2}\left[1+\cos \frac{\pi z}{L_{0}}\right] & -\mathrm{z}_{0} \leq \mathrm{z} \leq \mathrm{z}_{0} \\
1-m_{1}(z+L+d) & \mathrm{z}_{0}<\mathrm{z} \leq \mathrm{d} .
\end{array}\right.
$$

The analytical solutions of these equations are given by two methods. The first one uses regular perturbation technique, which based on small parameter $b_{1}{ }^{*}$ for small artery. The second one is based on utilizing the Homotopy perturbation technique.

\section{THE FIRST METHOD BY USING REGULAR PERTURBATION TECHNIQUE}

To solve the nonlinear system of (19)-(22) under the appropriate boundary conditions (23) and (24), we shall assume that any physical quantity, such as $w, P, T, C$, and $\mathrm{Q}$ may be represented as:

$$
\xi=\xi_{0}+b_{1}^{*} \xi_{1}+\ldots \ldots
$$

where $\xi_{0}$ is the zero order of $b_{1}^{*}$ and $\xi_{1}$ is the first one.

Substituting from (27) into the system of (19)-(24) and collect the terms of like powers of $b_{1}{ }^{*}$. This step generates zero and first order systems of partial differential equations with the corresponding boundary conditions. Because of the intricacy in treating these orders, we will investigate the solutions in case of small intestine or artery, in the light of the physical meaning of the length of the diameters of small arteries. Thus, we may presume that $r<<1$. Through the following subsections, we shall deem these orders:

\section{THE ZERO-ORDER SYSTEM}

Through the zero-order stage, the fluid behaves like a Newtonian fluid (in the absence of $b_{1}^{*}$ ). Therefore, the governing equations of motion will appear without the non-Newtonian parameter. As a result, one gets:

$\frac{d P_{0}}{d z}=\frac{1}{r} \frac{\partial}{\partial r}\left(r \frac{\partial w_{0}}{\partial r}\right)-N^{2} w_{0}$,

$\frac{1}{r} \frac{\partial}{\partial r}\left(r \frac{\partial T_{0}}{\partial r}\right)+\frac{D_{u} P_{r}}{1+R_{n}} \frac{1}{r} \frac{\partial}{\partial r}\left(r \frac{\partial C_{0}}{\partial r}\right)+\frac{B_{r}}{1+R_{n}}\left(\frac{\partial w_{0}}{\partial r}\right)^{2}+\frac{B_{r} N^{2}}{1+R_{n}} w_{0}^{2}=0$,

$\frac{1}{r} \frac{\partial}{\partial r}\left(r \frac{\partial C_{0}}{\partial r}\right)+\frac{S_{r} S_{c}}{r} \frac{\partial}{\partial r}\left(r \frac{\partial T_{0}}{\partial r}\right)-\alpha S_{c} C_{0}=0$,

$q_{0}=\int_{0}^{r_{2}} w_{0} r d r$,

$\hat{Q}_{0}=\frac{1}{\tau} \int_{0}^{\tau} Q_{0} d \tau$
In accordance with the boundary conditions:

$$
\begin{gathered}
=2 \pi \varepsilon \sin 2 \pi z, w_{0}=-1-\gamma^{*} \frac{\partial w_{0}}{\partial r}, \frac{\partial T_{0}}{\partial r}=-\beta_{t} T_{0}, \frac{\partial C_{0}}{\partial r}=-\beta_{c} C_{0} \\
r_{2}=r(z)+H_{1} \\
u_{0}=0, \frac{\partial w_{0}}{\partial r}=0, \frac{\partial T_{0}}{\partial r}=0, \frac{\partial C_{0}}{\partial r}=0 \text { at } r=0
\end{gathered}
$$

The solutions of (28)-(32) with the boundary conditions (33) and (34) are:

$$
\begin{aligned}
& Q_{0}=q_{0}+\frac{r_{2}^{2}}{2} \\
& \hat{Q}_{0}=q_{0}+\frac{1}{2}\left([R(z)]^{2}+\frac{\varepsilon^{2}}{2}\right) . \\
& G_{0}(z)=-\frac{4+2 \gamma^{*} N^{2} r_{2}(z)}{\gamma^{*}\left(r_{2}(z)\right)^{3}}\left(\hat{Q}_{0}-\frac{1}{2}\left([R(z)]^{2}+\frac{\varepsilon^{2}}{2}\right)+\frac{\left(r_{2}(z)\right)^{2}}{2+\gamma^{*} N^{2} r_{2}(z)}\right), \\
& w_{0}(r, z)=d_{1}(z)-\frac{G_{0}(z)}{N^{2}} \\
& T_{0}(r, z)=d_{13}(z) r^{4}+d_{14}(z) r^{2}+d_{15}(z),
\end{aligned}
$$

and

$$
C_{0}(r, z)=d_{11}(z) r^{2}+d_{16}(z)
$$

where $G_{0}(z)=\frac{d P_{0}}{d z}$ and $d_{1}, d_{2}, \ldots . ., d_{16}$ are calculated and known from the context.

\section{THE FIRST-ORDER SYSTEM}

The zero-order solutions acquired in the last subsection will be mingled through the first-order governed system to achieve non-homogeneous set of linear partial differential equations. This process will be recapitulated as follows:

$\frac{d P_{1}}{d z}=\frac{1}{r} \frac{\partial}{\partial r}\left(r\left(\frac{\partial w_{1}}{\partial r}+\left(\frac{\partial w_{0}}{\partial r}\right)^{n}\right)\right)-N^{2} w_{1}$,

$\frac{1}{r} \frac{\partial}{\partial r}\left(r \frac{\partial T_{1}}{\partial r}\right)+\frac{D_{u} P_{r}}{1+R_{n}} \frac{1}{r} \frac{\partial}{\partial r}\left(r \frac{\partial C_{1}}{\partial r}\right)+\frac{2 B_{r}}{1+R_{n}}$

$\left(\frac{\partial w_{1}}{\partial r} \frac{\partial w_{0}}{\partial r}+\left(\frac{\partial w_{1}}{\partial r}\right)^{n+1}\right)+\frac{B_{r} N^{2}}{1+R_{n}} w_{1}^{2}=0$,

$\frac{1}{r} \frac{\partial}{\partial r}\left(r \frac{\partial C_{1}}{\partial r}\right)+\frac{S_{r} S_{c}}{r} \frac{\partial}{\partial r}\left(r \frac{\partial T_{1}}{\partial r}\right)-\alpha S_{c} C_{1}=0$,

$q_{1}=\int_{0}^{r_{2}} w_{1} r d r$

$\hat{Q}_{1}=\frac{1}{\tau} \int_{0}^{\tau} Q_{1} d \tau$.

In accordance with the boundary conditions:

$$
\begin{aligned}
& u_{1}=0, w_{1}=-\gamma^{*}\left(\frac{\partial w_{1}}{\partial r}+\left(\frac{\partial w_{0}}{\partial r}\right)^{n}\right), \frac{\partial T_{1}}{\partial r}=-\beta_{t} T_{1}, \\
& \frac{\partial C_{1}}{\partial r}=-\beta_{c} C_{1} \text { at } r=r_{2}=r(z)+H_{1},
\end{aligned}
$$




$$
u_{1}=0, \frac{\partial w_{1}}{\partial r}=0, \frac{\partial T_{1}}{\partial r}=0, \frac{\partial C_{1}}{\partial r}=0 \text { at } r=0,
$$

The solutions of (41)-(45) with the boundary conditions (46) and (47) are:

$$
\begin{gathered}
Q_{1}=q_{1}+\frac{r_{2}^{2}}{2} \\
\hat{Q}_{1}=q_{1}+\frac{1}{2}\left([R(z)]^{2}+\frac{\varepsilon^{2}}{2}\right) . \\
G_{1}(z)=\frac{16}{\left(r_{2}(z)\right)^{2}\left(8 d_{32}(z)+\left(r_{2}(z)\right)^{2}\right)}\left[\left(\hat{Q}_{1}-\frac{1}{2}\left([R(z)]^{2}+\frac{\varepsilon^{2}}{2}\right)\right.\right. \\
\left.-\frac{\left(r_{2}(z)\right)^{2} d_{33}(z)}{2}-\frac{d_{19}(z)\left(r_{2}(z)\right)^{n+3}}{(n+1)^{2}(n+3)}\right] \\
w_{1}(r, z)=d_{33}(z)+\frac{d_{19}(z) r^{n+1}}{(n+1)^{2}}+\left(d_{32}(z)+\frac{r^{2}}{4}\right) G_{1}(z), \\
T_{1}(r, z)=d_{31}(z)+d_{30}(z)\left(\frac{d_{20}(z)}{4} r^{2}+\frac{d_{21}(z)}{16} r^{4}+\frac{d_{22}(z)}{36} r^{6}+\frac{d_{23}(z)}{(n+3)^{2}} r^{n+3}\right. \\
\left.+\frac{d_{22}(z)}{(n+5)^{2}} r^{n+5}+\frac{d_{25}(z)}{(2 n+4)^{2}} r^{2 n+4}\right),
\end{gathered}
$$

and

$$
\begin{aligned}
C_{1}(r, z)= & d_{28}(z)-\frac{S_{c} S_{r}}{d_{5}(z)\left(1+R_{n}\right)}\left(\frac{d_{20}(z)}{4} r^{2}+\frac{d_{21}(z)}{16} r^{4}+\right. \\
& \frac{d_{22}(z)}{36} r^{6}+\frac{d_{23}(z)}{(n+3)^{2}} r^{n+3} \\
& \left.+\frac{d_{24}(z)}{(n+5)^{2}} r^{n+5}+\frac{d_{25}(z)}{(2 n+4)^{2}} r^{2 n+4}\right)
\end{aligned}
$$

where $G_{1}(z)=\frac{d P_{1}}{d z}$ and $d_{i}, i=17,18, \ldots ., 33$, are known from the context.

From (37) and (50) the expression for the pressure gradient take the following form:

$$
\begin{aligned}
G(z) & =-\frac{4+2 \gamma^{*} N^{2} r_{2}(z)}{\gamma^{*}\left(r_{2}(z)\right)^{3}}\left(\hat{Q}_{0}-\frac{1}{2}\left([R(z)]^{2}+\frac{\varepsilon^{2}}{2}\right)+\frac{\left(r_{2}(z)\right)^{2}}{2+\gamma^{*} N^{2} r_{2}(z)}\right)+b_{1}^{*} \\
& \times\left[\frac { 1 6 } { ( r _ { 2 } ( z ) ) ^ { 2 } ( 8 d _ { 3 2 } ( z ) + ( r _ { 2 } ( z ) ) ^ { 2 } ) } \left[\left(\hat{Q}_{1}-\frac{1}{2}\left([R(z)]^{2}+\frac{\varepsilon^{2}}{2}\right)-\frac{\left(r_{2}(z)\right)^{2} d_{33}(z)}{2}\right.\right.\right. \\
& \left.\left.\left.-\frac{d_{19}(z)\left(r_{2}(z)\right)^{n+3}}{(n+1)^{2}(n+3)}\right)\right]\right],
\end{aligned}
$$

where $G_{1}(z)=\frac{d P}{d z}$.

From (38) and (51), the expression for the axial velocity component take the following form:

$$
\begin{aligned}
w(r, z)= & \left(d_{1}(z)-\frac{G_{0}(z)}{N^{2}}\right)+b_{1}^{*}\left[d_{33}(z)+\frac{d_{19}(z) r^{n+1}}{(n+1)^{2}}+\right. \\
& \left.\left(d_{32}(z)+\frac{r^{2}}{4}\right) G_{1}(z)\right] .
\end{aligned}
$$

The pressure rise and the friction force in the tube of length $L+d$, in their non -dimensional forms, are given by

$$
\begin{aligned}
\Delta P & =\int_{-L}^{d} G(z) d z \\
& =\int_{-L}^{-z_{0}} G(z) d z+\int_{-z_{0}}^{z_{0}} G(z) d z+\int_{z_{0}}^{d} G(z) d z, \\
\Delta F & =\int_{-L}^{d} r_{2}^{2}(-G(z)) d z \\
& =\int_{-L}^{-z_{0}} r_{2}^{2}(-G(z)) d z+\int_{-z_{0}}^{z_{0}} r_{2}^{2}(-G(z)) d z \\
& +\int_{z_{0}}^{d} r_{2}^{2}(-G(z)) d z,
\end{aligned}
$$

Because of the intricacy in evaluating these integrations, the values of them are estimated numerically.

In case of ignoring these considerations $\left(S, B_{0}, \gamma^{*}\right.$, $b_{1}{ }^{*}, h$ and $\phi$ tend to zero and $k_{1}$ tends to $\infty$ ), the pressure rise and friction force results good agreement with give the previous work obtained by Shapiro et al. (1969).

\section{THE SECOND METHOD BY USING HOMOTOPY PERTURBATION TECHNIQUE}

The amalgamation between the homotopy method and perturbation method is called homotopy perturbation method (HPM). This method eliminates the obstacles exist in the traditional perturbation method and at the same time all the advantages stay the same. The HPM was first presented by He (1999). The HPM does not relay on a small parameter in the equation. Utilizing homtopy technique is established with an embedding parameter $p h \in[0,1]$ which is presumed as a small parameter. The HPM can be applied to the non-linear differential (19)(21) subject to boundary conditions (23) and (24) as follows (Nadeem \& Akbar 2010; Siddiqui et al. 2008).

$\left.H\left(w, p_{h}\right)=L(w)-L\left(w_{0 h}\right)+p_{h} L\left(w_{0 h}\right)+p_{h}\left[\frac{1}{r} \frac{\partial}{\partial r}\left(b_{1}^{*} r\left(\frac{\partial w}{\partial r}\right)^{n}\right)\right)-\frac{d P}{d z}-N^{2} w\right],($

$$
\begin{aligned}
& H\left(T, p_{h}\right)=L(T)-L\left(T_{0 h}\right)+p_{h} L\left(T_{0 h}\right)+p_{h}\left[\frac{D_{u} P_{r}}{1+R_{n}} \frac{1}{r} \frac{\partial}{\partial r}\left(r \frac{\partial C}{\partial r}\right)+\frac{B_{r}}{1+R_{n}}\left(\left(\frac{\partial w}{\partial r}\right)^{2}+b_{1}^{*}\left(\frac{\partial w}{\partial r}\right)^{n+1}+\right.\right. \\
& \left.\left.N^{2} w^{2}\right)\right], \\
& H\left(C, p_{h}\right)=L(C)-L\left(C_{0 h}\right)+p_{h} L\left(C_{0 h}\right)+p_{h}\left[\frac{S_{r} S_{C}}{r} \frac{\partial}{\partial r}\left(r \frac{\partial T}{\partial r}\right)-\alpha S_{c} C\right] .
\end{aligned}
$$

For the appropriateness of this paper, the linear operator $L$ may be taken as

$L=\frac{1}{r} \frac{\partial}{\partial r}\left(r \frac{\partial}{\partial r}\right)$. The initial guesses $w_{0 h}, T_{0 h}$ and $C_{0 n}$ can be defined as

$$
\begin{aligned}
& w_{0 h}(r, z)=\frac{1}{4}\left(r^{2}-r_{2}^{2}(z)\right)-1-\gamma^{*}\left(\frac{r_{2}(z)}{2}+b_{1}^{*}\left(\frac{r_{2}(z)}{2}\right)^{n}\right), \\
& \text { and } \\
& \qquad T_{0 h}(r, z)=\frac{1}{4}\left(r^{2}-r_{2}^{2}(z)\right)-\frac{r_{2}(z)}{2 \beta_{t}} \text {, }
\end{aligned}
$$

$$
C_{0 h}(r, z)=-S_{c} S_{r}\left(\frac{r^{2}-r_{2}^{2}(z)}{4}-\frac{r_{2}(z)}{2 \beta_{c}}\right) .
$$


The solution $w_{0 h}(r, z)$ represents the velocity distribution in case of original flow (i.e. the flow before the peristalsis occurs) which is assumed to be local Hagen-Poiseuille flow.

The basic presumption is that the solutions of (58)(60) can be extended as a power series in $p_{h}$ :

$$
\begin{aligned}
& w(r, z)=w_{0 h}+p_{h} w_{l h}+\ldots, \\
& T(r, z)=T_{0 h}+p_{h} T_{l h}+\ldots,
\end{aligned}
$$

and

$$
C,(r, z)=C_{0 h}+p_{h} C_{1 h}+\ldots,
$$

Applying the same technique as done by Nadeem and Akbar (2010); and Siddiqui et al. (2008), the solutions of axial velocity, temperature and concentration for $p_{h}=1$, can be written as, respectively:

$$
\begin{aligned}
& w(r, z)=\frac{N^{2} f_{1}}{4} r^{2}+\frac{N^{2}}{64} r^{4}-\frac{2 b^{*}}{n+1}\left(\frac{r}{2}\right)^{n+1}+\left(\frac{r^{2}}{4}+f_{6}\right) G(z)+f_{9}, \\
& G(z)=\frac{16}{\left(r_{2}(z)\right)^{2}\left(8 f_{6}(z)+\left(r_{2}(z)\right)^{2}\right)}\left[\left(\hat{Q}-\frac{1}{2}\left([R(z)]^{2}+\frac{\varepsilon^{2}}{2}\right)-\frac{f_{9}}{2} r_{2}(z)\right.\right. \\
& \left.\left.-\frac{N^{2}\left(r_{2}(z)\right)^{4} f_{1}(z)}{16}-\frac{N^{2}\left(r_{2}(z)\right)^{6}}{384}+\frac{8 b^{*}}{(n+1)(n+3)}\left(\frac{r_{2}(z)}{2}\right)^{n+3}\right)\right], \\
& w(r, z)=\frac{N^{2} f_{1}}{4} r^{2}+\frac{N^{2}}{64} r^{4}-\frac{2 b^{*}}{n+1}\left(\frac{r}{2}\right)^{n+1}+\left(\frac{r^{2}}{4}+f_{6}\right) G(z)+f_{9}, \\
& T(r, z)=-\frac{\beta_{r}}{1+R_{n}}\left(\frac{N^{2} f_{1}^{2}}{4} r^{2}+\frac{\left(1+N^{2} f_{1}\right)}{32} r^{4}+\frac{N^{2}}{480} r^{5}+\frac{4 b^{*}}{(n+3)^{2}}\left(\frac{r}{2}\right)^{n+3}\right) \\
& +\frac{D_{u} P_{r}}{4\left(1+R_{n}\right)} r^{2}+f_{10}
\end{aligned}
$$

and

$$
C(r, z)=-\frac{\alpha S_{c}^{2} S_{r}}{64} r^{4}+\left(\frac{\alpha S_{c} f_{3}-S_{c} S_{r}}{4}\right) r^{2}+f_{11}
$$

where $G(z) \frac{d P}{d z}=$ and $f_{j}, j=1,2, \ldots ., 11$, are known from the context

The expressions of the pressure rise and the friction force in the tube of length $L+d$, in this case, are computed numerically as mentioned before.

\section{RESULTS AND DISCUSSION}

This section deals with the calculation of numerical estimations. It is convenient to divide these calculations into two categories, as follows:

\section{PUMPING CHARACTERISTICS}

In order to identify the quantitative effects of several parameters on the obtained distributions of the axial velocity $w$, temperature $T$, concentration $C$, pressure rise $\underline{\Delta} P$ and friction force $\underline{\Delta} F$, the mathematical software (Mathematica) is used. The considered ranges of governing parameters are taken from related studies (Eldabe 2016; Fung \& Yin 1969; Verma \& Parihar 2010).
The curves $I$ refer to studying the effects of various parameters in case of the first method of solution by using regular perturbation technique. However, the curves II represent the influences of the parameters in case of the second method of solution by utilizing the homotopy perturbation technique. Some essential results are graphically exhibited in Figures 2-9 as follows.

Figure 2(A) illustrates the variation of axial velocity for different values of Hall parameter $m$. This figure shows the increasing effect of axial velocity for increasing Hall parameter $m$. Actually, for larger values of $m$, the effective conductivity decreases. It leads to the decrease in magnetic damping force. Therefore, the axial velocity is enhanced. Figure 2(B) indicates the variation of axial velocity $w(z)$ for different values of the Hartmann number $H^{2}$. It is shown that the axial velocity decreases with the increase of $H^{2}$. This phenomenon is conforming to the theory which states that the increase in Hartmann number causes increase in the Lorentz force. It is recognized that Lorentz force hinders the flow. This illustrates that if we increase the strength of magnetic field, the flow of blood will be hindered. Figure 2(C) shows the variation of axial velocity, $w(z)$ for different values of permeability parameter $k_{1}$. It is observed that the axial velocity increases with the increase of $k_{1}$. An increasing of $k_{1}$ leads to minimize the drag force. Therefore, this is lead to increase the flow velocity. Figure 2(D) shows the variation of axial velocity $w(z)$ for different values of power index $n$. The features of shearthinning and shear-thickening fluids are entirely different from a Newtonian fluid. It is indicated that, in case of shear thickening fluid $n>1$, the values of the axial velocity is greater than those in case of shear thinning behavior $n<1$ and also for Newtonian behavior $n=1$. Moreover, this result conforms to the result of Khan et al. (2010). Furthermore, Figure 2(A), 2(B), 2(C) and 2(D) illustrates that the values of velocity in case of using regular perturbation technique () is higher than those in case of utilizing the homotopy perturbation technique (II).

Figure 3(A) shows the variation of axial velocity $w(z)$ for different values of maximum height of stenosis $h$. It is indicated that if the domain of the maximum height of stenosis $h$ becomes $(-0.5 \leq z \leq 0.5)$, the axial velocity decreases with the increase of $h$. Reciprocally, the curves of this velocity are coincide to each other's. It is also found that, in case of no-stenosis $(h=0)$, the values of the axial velocity is higher than that in case of stenosis. Thus, for the diseases of blood clot, the existence of the clots, at the artery straitens the blood flow, leads to a harmful effect on the body organs (Nichols \& Orourke 1973). Figure 3(B) indicates the variation of axial velocity $w(z)$ for different values of slip parameter $\gamma^{*}$. It is shown that the velocity depresses with increase of $\gamma^{*}$. Figure 3(C) illustrates the variation of axial velocity $w(z)$ for different values of the flow rate $Q$ in case of the homotopy technique. It is observed that the axial velocity increases by the increasing of $Q$. 
Also, this figure indicates the special case of peristaltic transport of Newtonian fluid which refer to ignoring the parameters $m=0, B_{0}=0$, (absence of magnetic field), $H^{2}=0, \gamma^{*}$ (non slip conditions), $k_{1}$ tends to $\infty$ (absence of porous media), $\phi=0$ (no-tapered ) $b^{*}=0$ and $h=0$ (no-stenosis). Throughout this figure, we have recorded the previous results which have been early obtained by Fung and Yin (1969). Furthermore, it is found out that the value of the axial velocity is lower than that in case of Newtonian fluid. Figure 3(D) shows a comparison between the solution in case of using regular perturbation technique (I), the previous work of El-dabe et al. (2016) ( $m=0$ and $H^{2}=0$ ) and the case of peristaltic transport of Newtonian fluid (Fung \& Yin 1969). It is observed that the values of axial velocity in case of Newtonian fluid are higher than those in the other two cases. Also, it was found out that the values of axial velocity in case of $m$ $=0$ and $H^{2}=0$ are higher than those in case of studying.

Figure 4(A) shows the variation of axial velocity $w$ vs radial distance $r$ for different values of the taper angle $\phi$. The significance of the impact of vessel tapering with the shape of stenosis requires particular concern. Also, the tapering has a considerable aspect in the arterial system (Verma \& Parihar 2010). Therefore, our interest is concentrated on investigating the flow through a tapered tube with stenosis. It is shown that in case of the diverging tapered artery $\phi=0.05(>0)$, the values of the axial velocity are greater than those in case of the non tapered artery $\phi=0$ and the convergent tapered one $\phi=$ $0.05(<0)$. Figures $4(\mathrm{~B})$ illustrates the variation of axial velocity $w(r)$ for different values of the Sisko fluid parameter $b^{*}$. It refers to the ratio of a power-low part to a viscous part in a Sisko fluid if $(n \neq 1)$. The case of ( $n$ $\neq 1, b_{1}{ }^{*}=0$ ) shows a viscous Newtonian fluid. However, the case of $\left(b^{*} \rightarrow \infty\right)$ describes a purely power-low model (Mekheimer \& El Kot 2012). It is shown that the axial velocity decreases with the increase of $b^{*}$ because of the rise in Sisko fluid parameter $b^{*}$ leads to rise the elasticity in the fluid. That makes the fluid thicker and decreases its velocity. Moreover, the impact of this parameter complies with the previous work of El-dabe et al. (2016), and Mekheimer and El Kot (2012). Furthermore, Figure 4(A) and 4(B) demonstrates that the values of velocity in case of using the homotopy perturbation technique (II) is lower than those in case of utilizing the regular perturbation technique $(I)$.

The variation of temperature profile $T(r)$ for different values the radiation parameter $R_{n}$ is shown in Figure 5(A). It is shown that an increase in radiation parameter $R_{n}$ leads to the decrease of the fluid temperature. Figure $5(\mathrm{~B})^{n}$ describes the variation of temperature $T(r)$ for different values of the heat transfer Biot number $\beta_{t}$. It is found out that the temperature decreases with the increase of $\beta$. The obtained result shows that the thermal conductivity of the fluid decreases with increase of $\beta_{t}$ and that leads to decrease in temperature. The variation of temperature profile $T(r)$ for different values of the Dufour number $D_{u}$ and Soret number $S_{r}$ is illustrated in Figure
5(C). It is noticed that temperature enhances with the increase of both $D_{u}$ and $S$. Actually, The increase of both both $D_{u}$ and $S_{r}$ leads to the rise of thermal conductivity, consequently, the temperature is enhanced. Physically, the diffusion-thermo or Dufour effect known as a heat flux conducted when a chemical system undergoes a concentration gradient. These influences relied on thermal diffusion which is though very small. However, these influences become fundamental sometimes due to the different molecular weights. Mass diffusion pursues by the disparate distribution of species producing a concentration gradient. Moreover, a temperature gradient can be considered as a driving force for mass diffusion known as thermo-diffusion or Soret effect. Therefore, the increasing in Soret effect leads to increasing the temperature gradient. Furthermore, the impact of this parameter is corresponds to the previous work of (Hayat et al. 2016). Through Figure 5(D), the influence of the Brickmann number $B$ on temperature is illustrated. It can be noted that the dimensionless temperature increases with the increase of $B_{r}$ value. This situation can be analyzed by higher thermal energy which generated via the viscous dissipation. So, the fluid temperature increases. However, this behavior causes some unexpected problems such as shrinkage, stretching, and deterioration of the arteries. Additionally, the figures of temperature show that the values of temperature in case of using regular perturbation technique $(I)$ are higher than those in case of utilizing the homotopy perturbation technique (II).

The influence of chemical reaction on concentration is also significant. Figure 6(A) depicts the variation of concentration $C$ vs $r$-axis for different values of chemical reaction number $\alpha$. Chemical reaction enhances the interfacial mass transfer rate which leads to the decrease of the concentration. Figure 6(B) illustrates the variation of concentration $C$ vs $r$-axis for different values of the Schmidt number $S_{c}$. It is shown that the concentration profile decreases when $S_{c}$ increases. As Schmidt number is known as the ratio of momentum diffusivity (viscosity) to mass diffusivity. Thus, the increase of $S_{c}$ leads to the decrease of mass diffusion. This process enhances the inter-molecular force, consequently, causes a reduction in concentration. Moreover, these figures depict that the values of concentration in case of using regular perturbation technique $(I)$ is higher than those in case of utilizing the homotopy perturbation technique (II).

The pressure rise $\Delta P^{*}$ is plotted vs the mean flow rate for different values of the Hartmann number $H^{2}$, slip parameter $\gamma^{*}$, the maximum hight of the stenosis $h$ and the Hall current parameter $m$ in Figure 7(A) and 7(B), respectively. The peristaltic pumping occurs for $\left(\Delta P^{*}>\right.$ 0 . Also, the free pumping occurs for $\left(\Delta P^{*}=0\right)$. The copumping holds when $\left(\Delta P^{*}<0\right)$. It is also noticed that the peristaltic pumping region decreases as the $\gamma^{*}$ increase for both case of $(I)$ and $(I I)$. Moreover, it is found out that, for the free pumping case, there are no noticeable differences observed. In contrast, in the co-pumping region, the pumping rate increases by increasing the 
values of $\gamma^{*}$. It is found that in 'pumping region $\left(\Delta P^{*}>0\right)$ ', the pressure rise increases with the increase of Hartman number $H^{2}$. Physically, due to magnetic interactions into the flow, pressure rise per wave length upturns rapidly. It is noted that pressure rise gives the opposite results in the 'augmented region $\left(\Delta P^{*}<0\right)$ '. It is also showed that the pressure rise is an increasing function of the increase in the Hartmann number $H^{2}$ in case of second method of solution by utilizing the homotopy perturbation technique. Finally, the relation between pressure rise and mean flow rate is linear.

Figure $8(\mathrm{~A})$ and $8(\mathrm{~B})$ illustrates the variation of the friction force $\Delta F$ vs the mean flow rate for various values of the Hartmann number $H^{2}$ and slip parameter $\gamma^{*}$. It is noticed that the friction forces have counteractive demeanors when compared with the pressure rise.
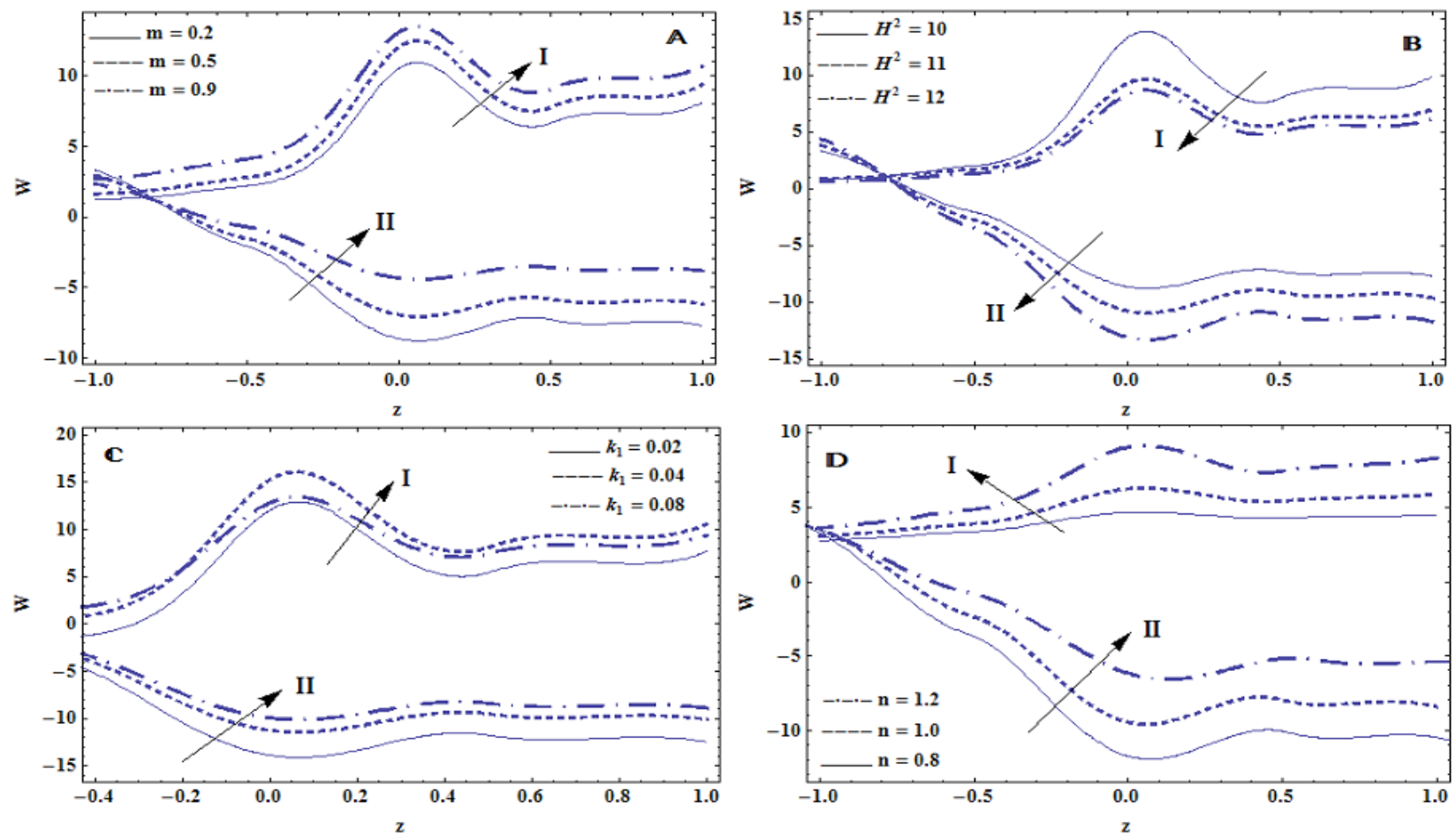

FIGURE 2. Variation of the axial velocity $w(z)$ for different values of $m, H^{2}, k_{1}, n$
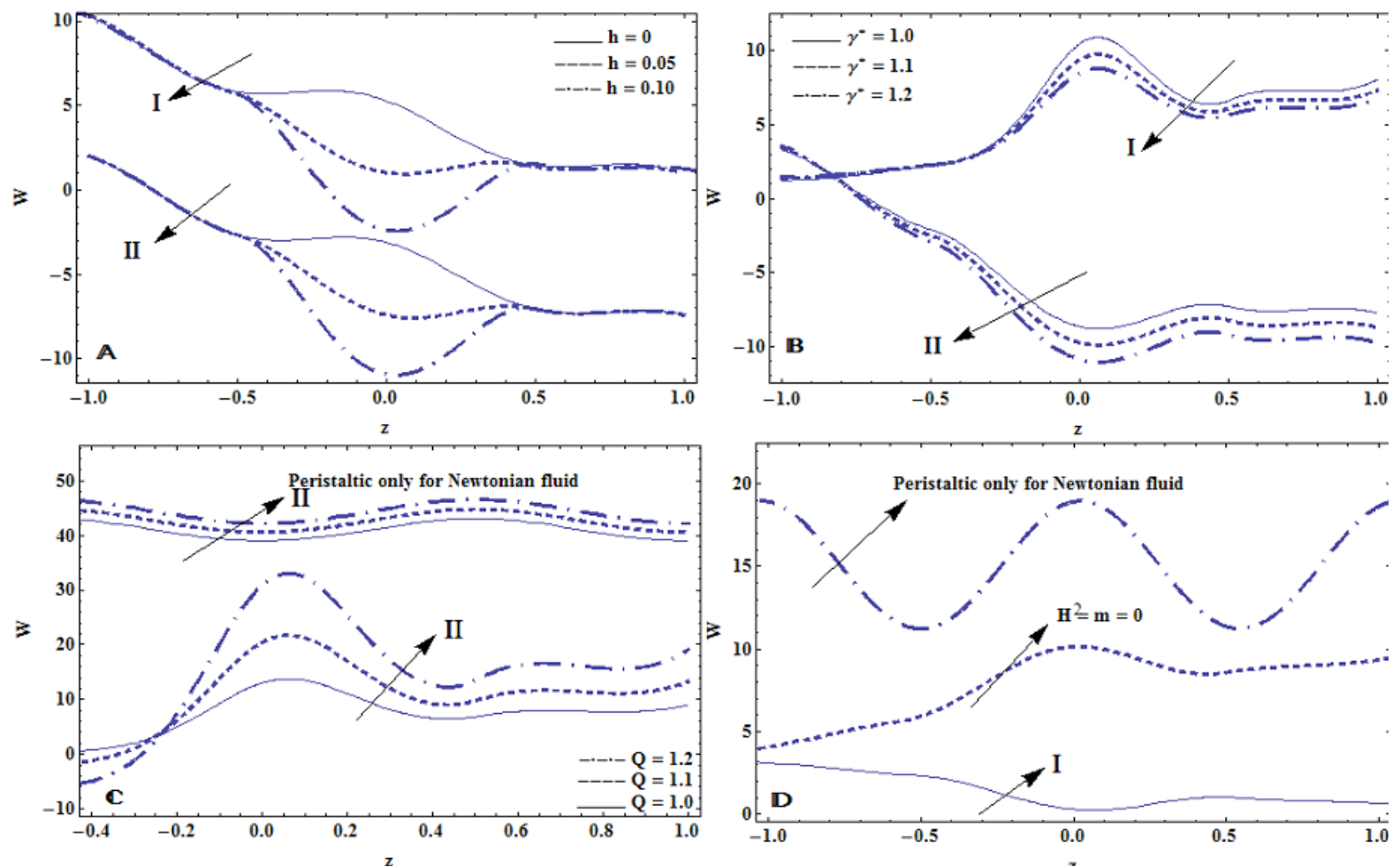

FIGURE 3. Variation of the axial velocity $w(z)$ for different values of $h, \gamma^{*}, Q$ and special case 

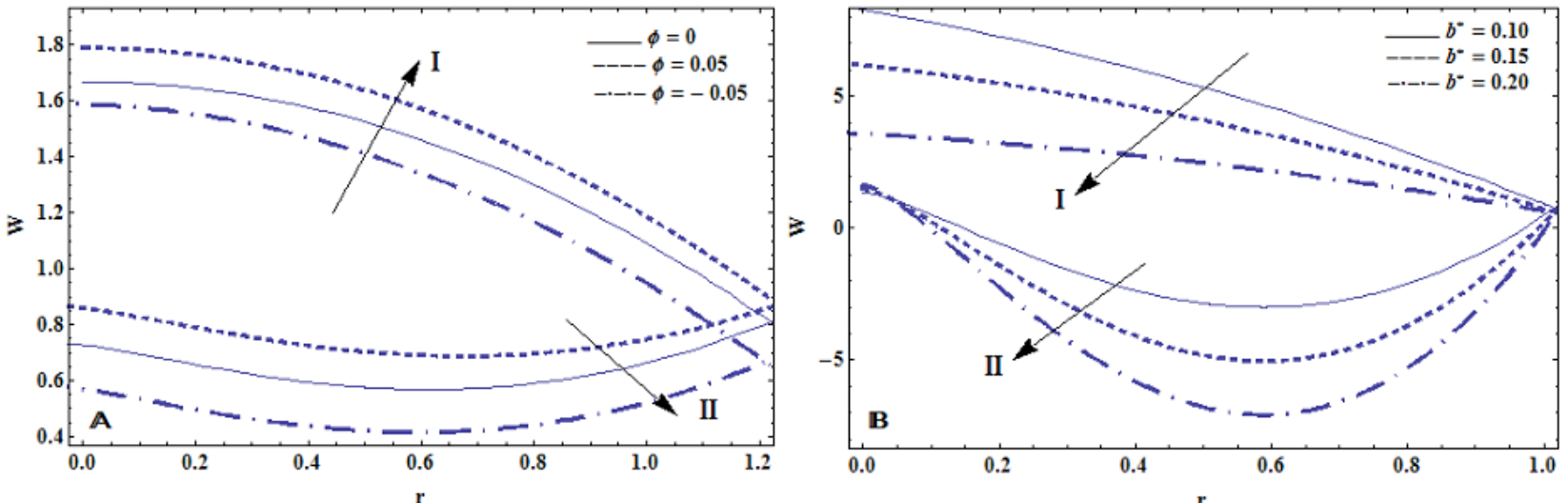

FIGURE 4. Variation of the axial velocity $w(r)$ for different values of $\phi$ and $b^{*}$
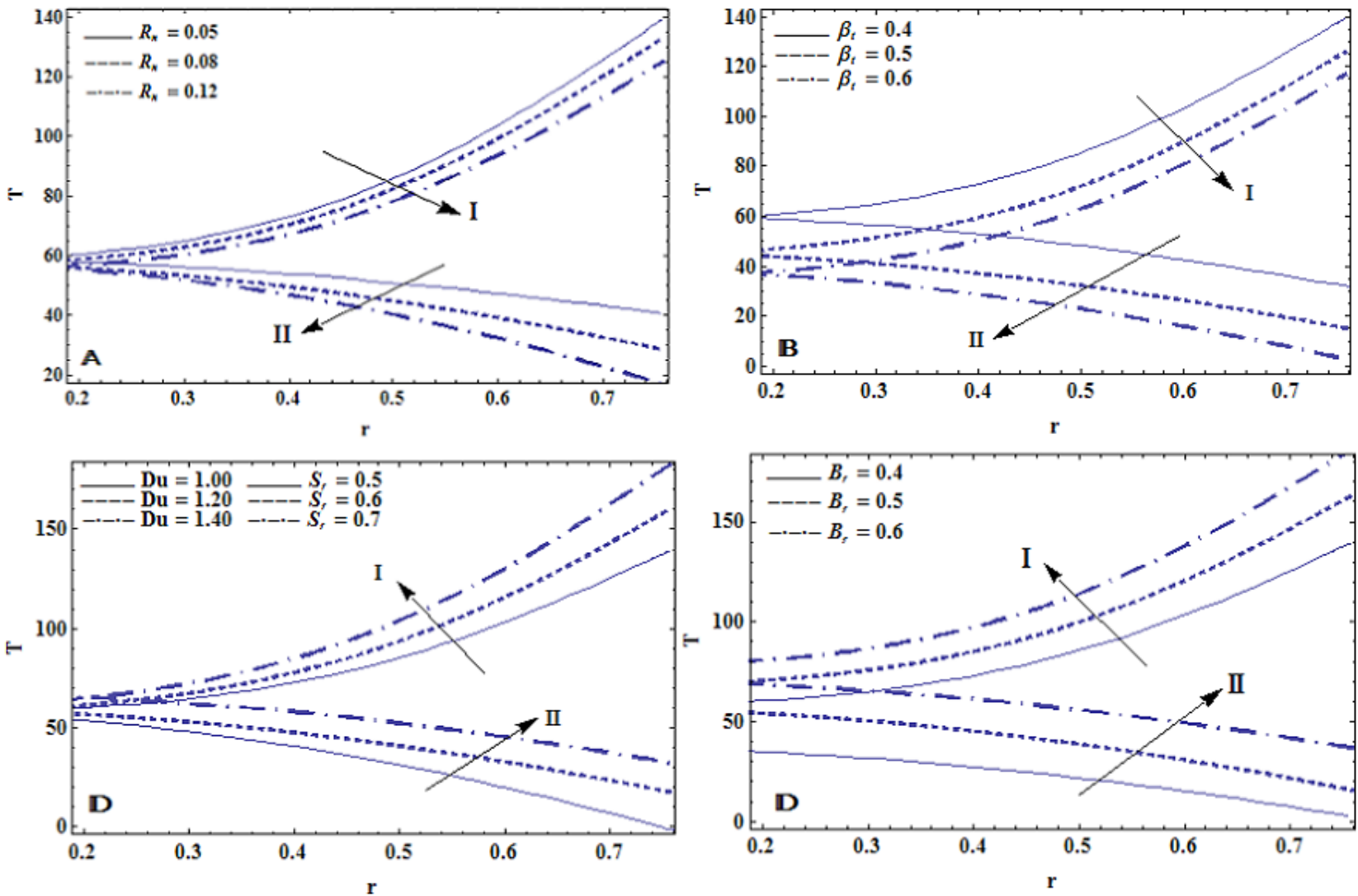

FIGURE 5. Variation of the temperature distribution $T(r)$ for different values of $R_{n}, \beta_{t}, D_{u}, S_{r}$ and $B_{r}$
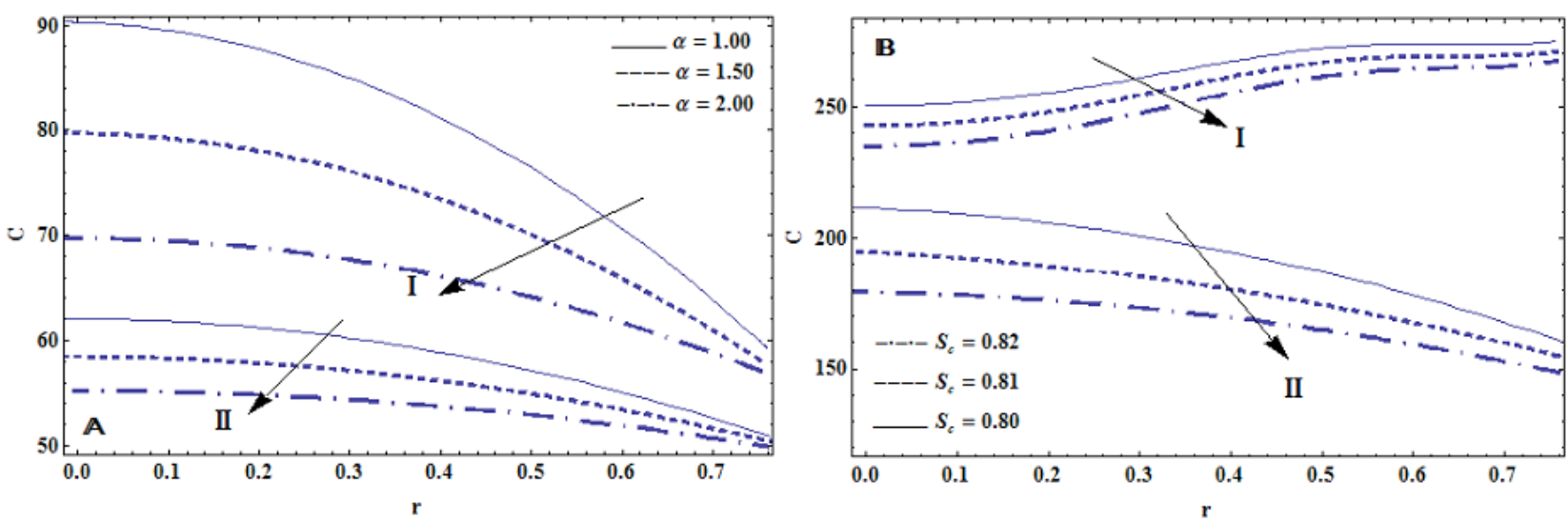

FIGURE 6. Variation of the concentration $C$ for different values of $\alpha$ and $S$ 

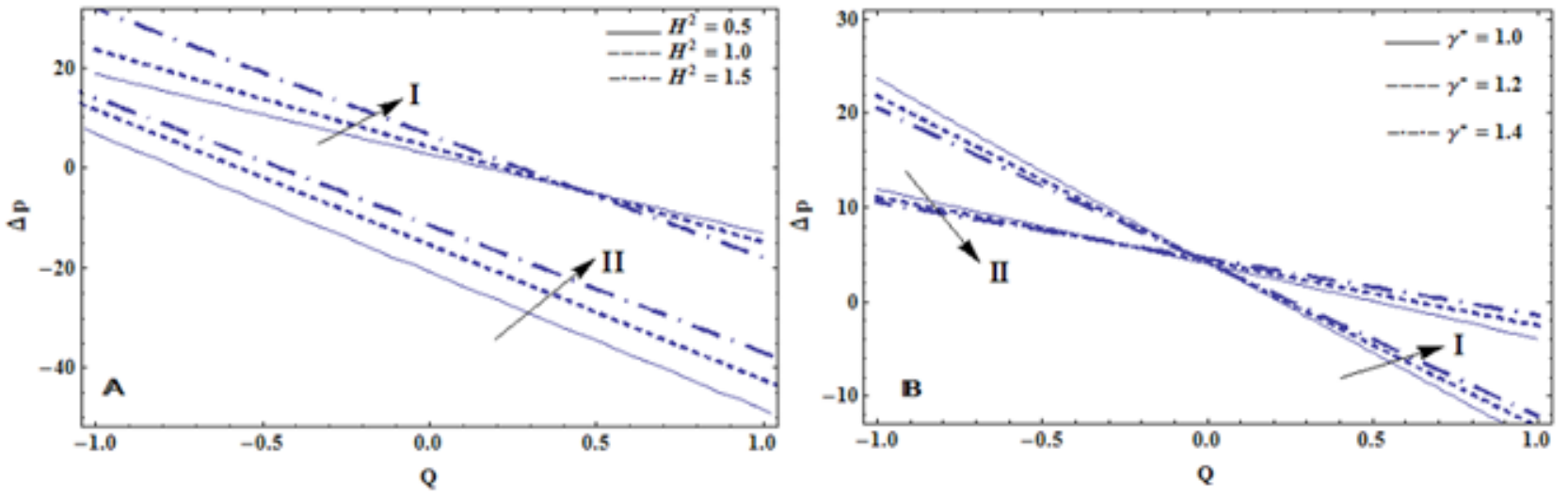

FIGURE 7. Variation of pressure rise $\Delta P^{*}$ vs mean flow rate for different values of $\gamma^{*}$ and $H^{2}$
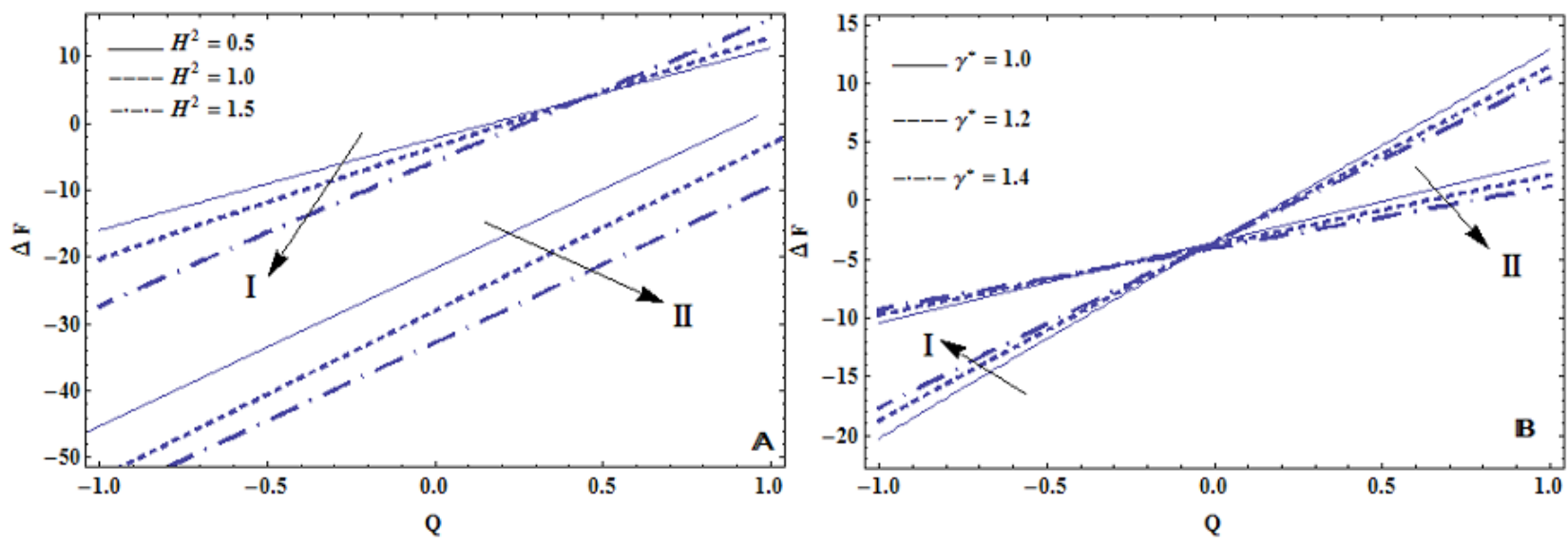

FIGURE 8. Variation of friction force on the outer tube $\Delta F$ vs mean flow rate for different values of $\gamma^{*}$ and $H^{2}$
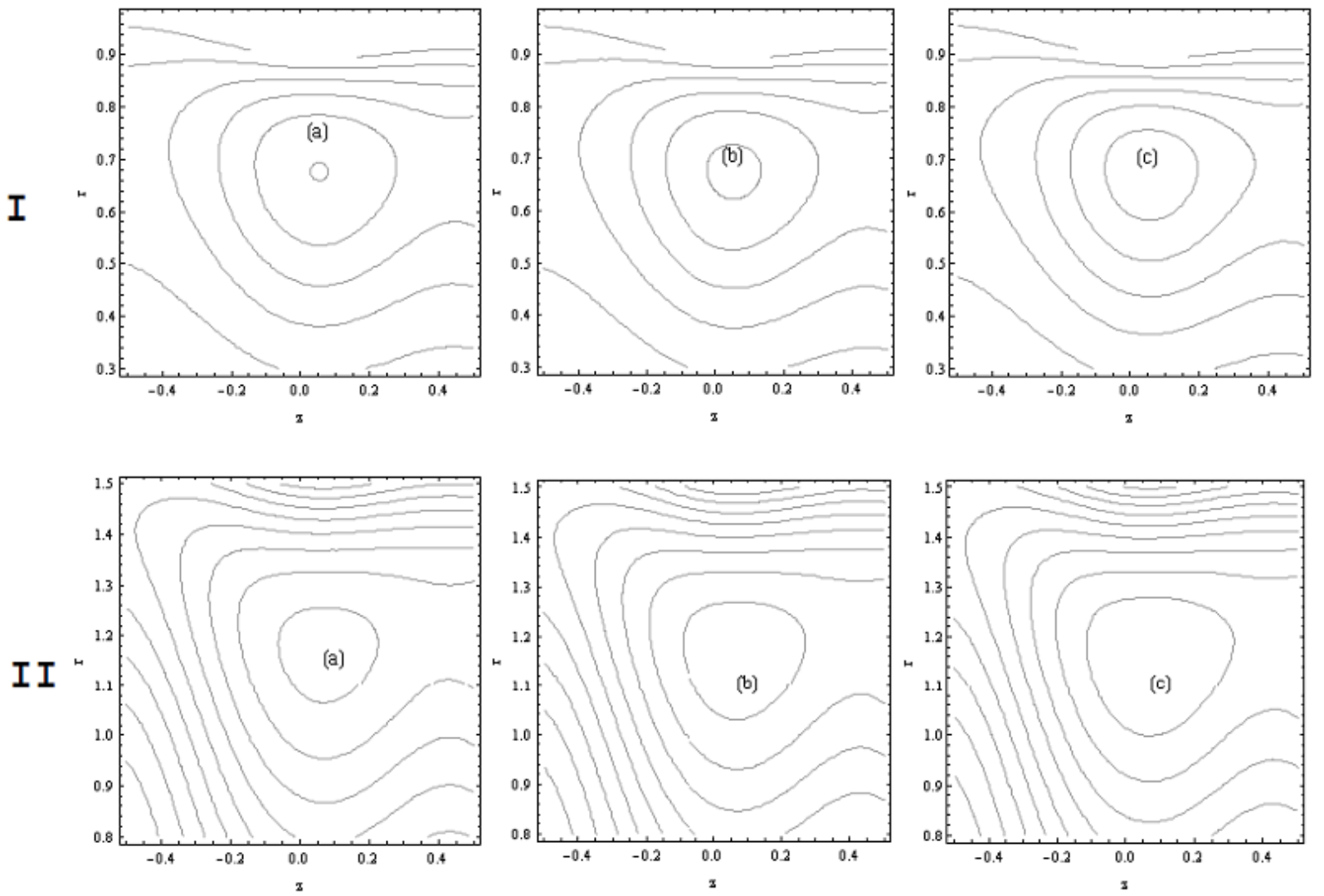

FIGURE 9. Streamlines for different values of the Hall current parameter $m$ 

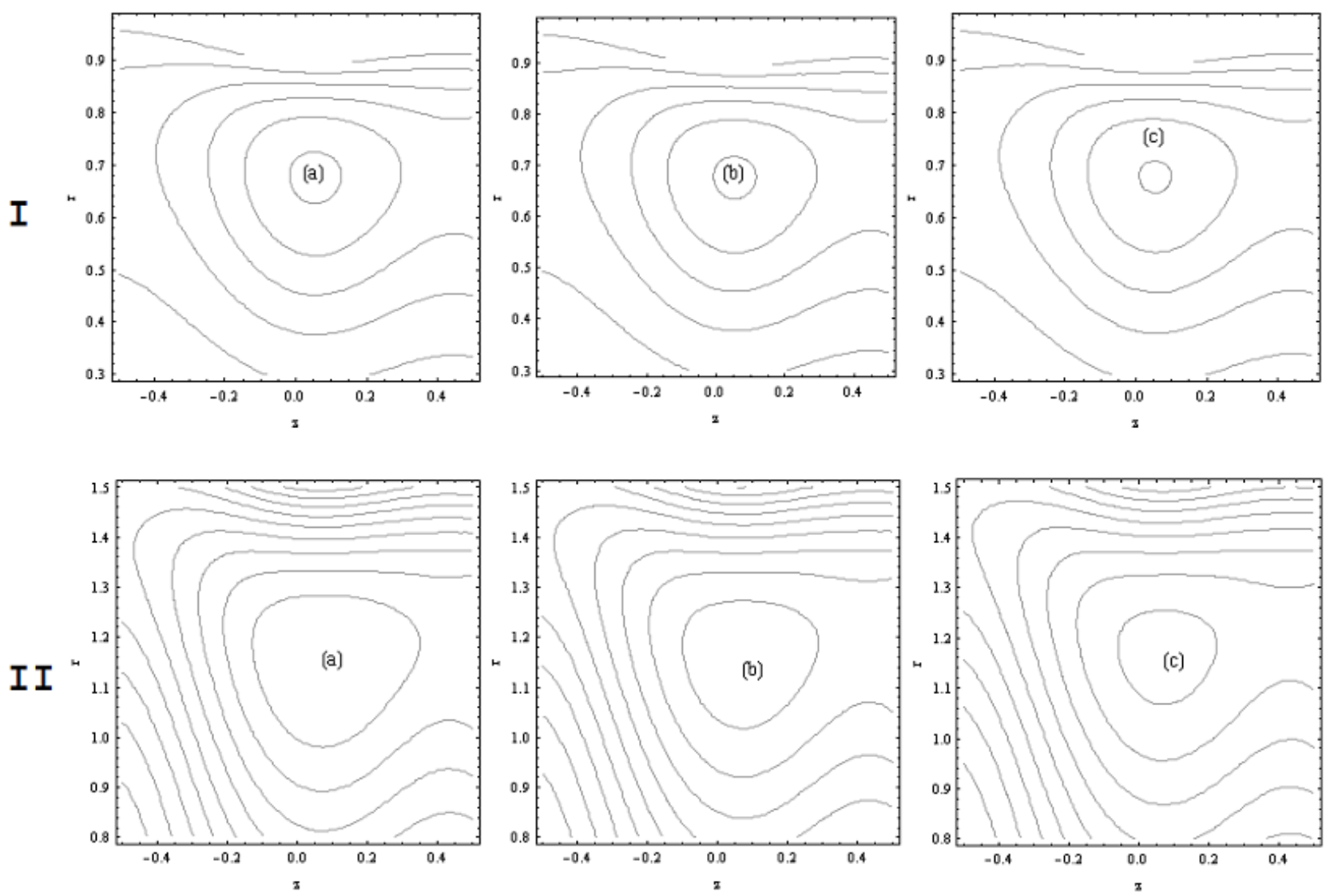

FIGURE 10. Streamlines for different values of the Hartmann number $H^{2}$
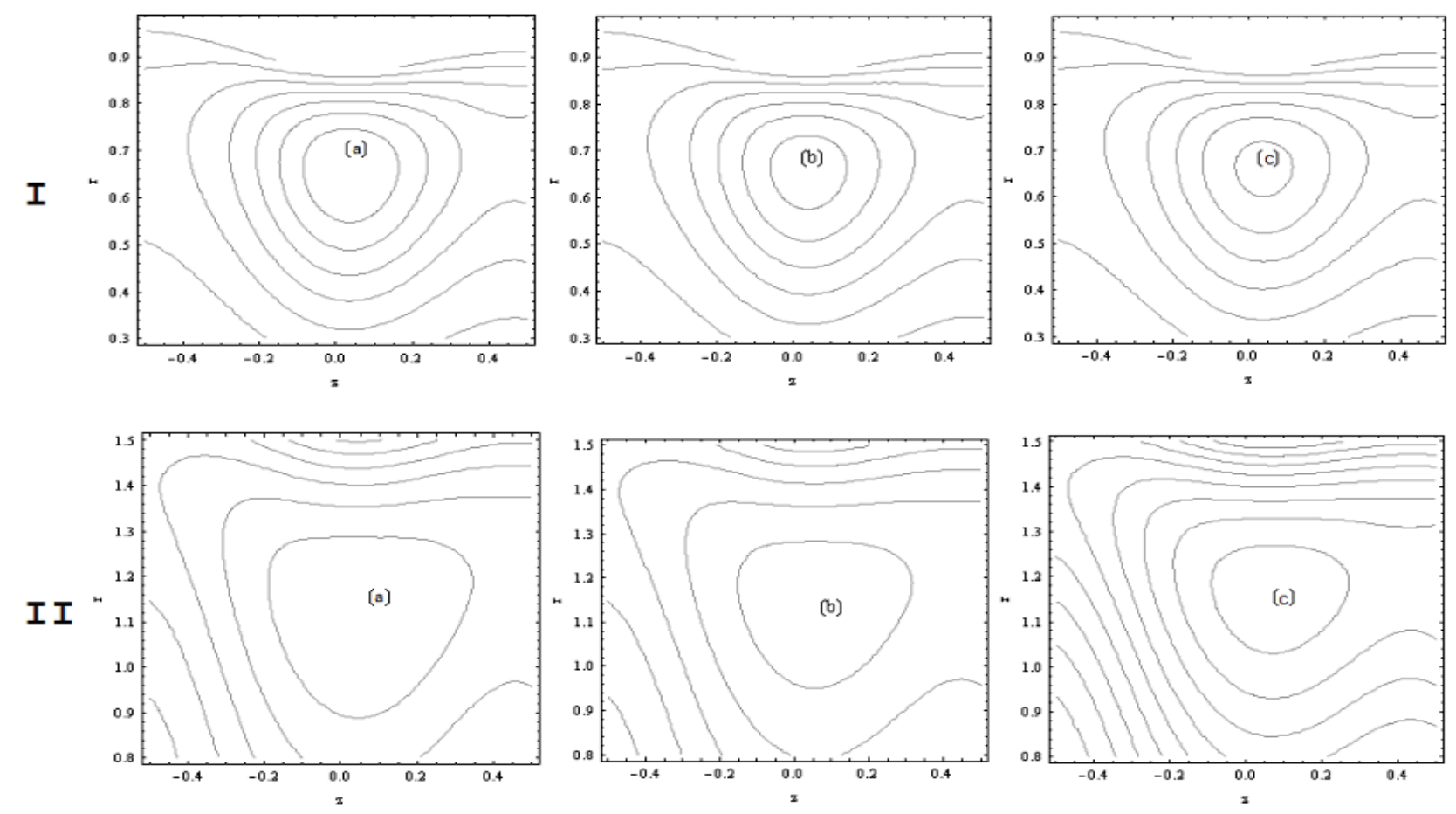

FIGURE 11. Streamlines for different values of maximum height of stenosis $h$ 


\section{TRAPPING}

Trapping is an essential physical procedure of peristaltic transmission. Trapping occurs only in particular states. As shown in the wave frame of reference, a set of streamlines may be showed in the most expanded section of the cylinder. This set of streamlines includes a bolus of fluid that moves with the wave in the laboratory frame. There is an inner circulation existent inside the bolus. However, all the contained fluid particles move ahead along with a mean velocity which is equal to the wave speed. Therefore, the structure of an internally circulating bolus of the fluid is known as a trapping. As well, bolus is determined as a volume of fluid bounded by closed streamlines. In addition, the trapping phenomenon has been discussed by many researchers, such as Jaffrin (1973) and Shapiro et al. (1969).

The following figures depict the stream lines graphs for various values of several parameters. The influence of the Hall current parameter $m$ on trapping is shown in Figure 9. It is illustrated that the trapped bolus increases in size by the increase of the Hall current parameter $m$. Indeed, by the increase of $m$, the effective conductivity decreases. It causes a reduction in magnetic damping force. Therefore, the size of the trapped bolus increased. The influence of the Hartmann number $H^{2}$ on the trapping are displayed in Figure 10. It is observed that the bolus decreases in size by the increasing of $H^{2}$. Actually, it is known that the increase in Hartman number causes an increase in the Lorentz force. Hence, blood flow becomes slow and leads to decreasing the size of the trapping bolus. Figure 11 displays the effects of the maximum height of stenosis $h$ on the trapping. It is shown that the bolus reduces in size by the increasing of $h$. Because of increasing the maximum height of stenosis, the arteries become narrower. Thus, the blood movement becomes slower in arteries and that causes a reduction in the size of trapping bolus. From the previous figures, it is noted that the size of trapped bolus in case of the second method of solution by utilizing the homotopy perturbation technique (II) is larger than those in case of the first method of solution by using regular perturbation technique $(I)$.

\section{CONCLUSION}

This study provides a theoretical approach is offered to construe the actions of the Hall current and Joule heating effects on peristaltic transport of blood flow. The flow is inflowing via a tapered artery with mild stenosis. The procedure is influenced by an external uniform magnetic field. The blood is represented by a Sisko model. Furthermore, the effects of slip condition for velocity and porous medium are investigated. Also, convective conditions for heat and mass transfer are considered. Moreover, the impacts of radiative heat transfer and chemical reactions are taken into account. The combined influences of viscous dissipation and radiation in energy expression are discussed. Also, Soret and Dufour features are investigated. The governing equations of motion, energy and concentration are analytically solved by utilized the long wavelength and low Reynolds number approximations. These equations are solved by two methods. The first one depends on the regular perturbation technique, which based on small wave number for small artery, while the second one is based on utilizing the Homotopy perturbation technique. The distributions of velocity, stream function, temperature and concentration are acquired. The formulations of pressure rise and friction force are obtained in terms of dimensionless flow rate $Q$ by depending on the numerical integration. The numerical calculations are presumed to obtain the impacts of several parameters, such as Hall current parameter $m$, Dufour number $D_{u}$, the taper angle $\phi$, Hartmann number $H^{2}$, Schmidt number $S_{c}$, the Biot number $\beta$, and chemical reaction number $\alpha$, on the above distributions. Trapping phenomena is also discussed. It is found out that the axial velocity increases with the increase of both $m, k_{1}$ and by the decrease of both $S$ and $H^{2}$. Also, the axial velocity decreases with the increase of $h$ in the region of mild stenosis. It is noted that the values of velocity in case of using regular perturbation technique $(I)$ is higher than those in case of utilizing the homotopy perturbation technique $(I I)$.

Finally, this study has many applications in many scientific areas such as medicine. The description of blood flow within the arteries in the presence of blood clots represents the study case. Thus, the study is considered to be very fundamental in the field of biofluid mechanics.

\section{ACKNOWLEDGEMENTS}

The authors are very thankful to the editor and referees for their valuable comments and suggestions.

\section{REFERENCES}

Abdelsalam, S.I. \& Bhatti, M.M. 2019. New insight into AuNP applications in tumour treatment and cosmetics through wavy annuli at the nanoscale. Scientific Reports 9(260): 1-14.

Abdelsalam, S.I. \& Bhatti, M.M. 2018. The study of nonNewtonian nanofluid with hall and ion slip effects on peristaltically induced motion in a non-uniform channel. RSC Advances 8: 7904-7915.

Ang, K.C. \& Mazumdar, J.N. 1997. Mathematical modeling of three dimensional flow through an asymmetric arterial stenosis. Mathematical and Computer Modelling 25(1): 19-29.

Arora, C.P. 1997. Heat and Mass Transfer. 2nd edition. Delhi: Khanna Publishers.

Bhatti, M.M., Zeeshan, A. \& Ellah, R. 2016. Simultaneous effects of coagulation and variable magnetic field on peristaltically induced motion of Jeffrey nanofluid containing gyrotactic microorganism. Microvascular Research 110: 32-42.

Burns, J.C. \& Parkes, T. 1967. Peristaltic motion. Journal of Fluid Mechanics 29(4): 731-743.

Chakravarty, S. \& Sen, S. 2005. Dynamic response of heat and mass transfer in blood flow through stenosed bifurcated arteries. Korea-Australia Rheology Journal 17(2): 47-62.

Chakravarty, S., Datta, A. \& Mandal, P.K. 1995. Analysis of nonlinear blood flow in a stenosed flexible artery. 
International Journal of Engineering Science 33(12): 18211837.

Di Federico, V., Longo, S., King, S.E., Chiapponi, L., Petrolo, D. \& Ciriello, V. 2017. Gravity driven flow of Herschel-Bulkley fluid in a fracture and in a 2D porous medium. Journal of Fluid Mechanics 821: 59-84.

El-dabe, N.T. \& Abou-zeid, M. 2014. MHD peristaltic flow with heat and mass transfer of micropolar biviscosity fluid through a porous medium between two co-axial tubes. Arabian Journal for Science and Engineering 39(6): 50455062.

El-dabe, N.T., Moatimid, G.M., Hassan, M.A. \& Mostapha, D.R. 2016. Effect of partial slip on peristaltic flow of a Sisko fluid with mild stenosis through a porous medium. Applied Mathematics \& Information Sciences 10(2): 1-15.

El-dabe, N.T., Hassan, M.A. \& Abou-zeid, M. 2015. Wall properties effect on the peristaltic motion of a coupled stress fluid with heat and mass transfer through a porous medium. Journal of Engineering Mechanics 142(3): 04015102.

El-dabe, N.T., Kamel, K.A., Galila Abd-Allah, M. \& Ramadan, S.F. 2013. Heat absorption and chemical reaction effects on peristaltic motion of micropolar fluid through a porous medium in the presence of magnetic field. African Journal of Mathematics and Computer Science Research 6(5): 94-101.

Eldabe, N.T., El-Sayed, M.F., Ghaly, A.Y. \& Sayed, H.M. 2008. Mixed convective heat and mass transfer in a nonNewtonian fluid at a peristaltic surface with temperaturedependent viscosity. Archive of Applied Mechanics 78: 599-624.

Eldabe, N.T., Ghaly, A.Y. \& Sayed, H.M. 2007. MHD Peristaltic flow of non-Newtonian fluid through a porous medium in circular cylindrical tube. Bulletin of the Calcutta Mathematical Society 99: 123-136.

Fung, Y.C. \& Yin, F. 1969. Peristaltic waves in circular cylindrical tubes. Journal of Applied Mechanics 36(3): 579-587.

Hayat, T., Javed, S., Khan, M., Imran Khan, M. \& Alsaedi, A. 2019. Physical aspects of irreversibility in radiative flow of viscous material with cubic autocatalysis chemical reaction. European Physical Journal Plus 134(4): 1-24.

Hayat, T., Aslam, N., Khan, M., Imran Khan, M. \& Alsaedi, A. 2018a. Physical significance of heat generation/absorption and Soret effects on peristalsis flow of pseudoplastic fluid in an inclined channel. Journal of Molecular Liquids 275: 599-615.

Hayat, T., Qayyum, S., Khan, M. \& Alsaedi, A. 2018b. Entropy generation in magnetohydrodynamic radiative flow due to rotating disk in presence of viscous dissipation and Joule heating. Physics of Fluids 30(1): 017101.

Hayat, T., Zahir, H., Alsaedi, A. \& Ahmad, B. 2017. Hall current and Joule heating effects on peristaltic flow of viscous fluid in a rotating channel with convective boundary conditions. Results in Physics 7: 2831-2836.

Hayat, T., Nawaz, S., Alsaedi, A. \& Rafiq, M. 2016a. Mixed convective peristaltic flow of water based nanofluids with Joule heating and convective boundary conditions. PLoS ONE 11(4): 1-28.

Hayat, T., Rafiq, M. \& Ahmad, B. 2016b. Soret and Dufour effects on MHD peristaltic flow of Jeffrey fluid in a rotating system with porous medium. PLOS ONE 11(1): 1-18.

Hayat, T., Iqbal, R., Tanveer, A. \& Alsaedi, A. 2016. Influence of convective conditions in radiative peristaltic flow of pseudoplastic nanofluid in a tapered asymmetric channel. Journal of Magnetism and Magnetic Materials 408: 168-176.
Hayat, T., Abbasi, F.M., Ahmad, B. \& Alsaedi, A. 2014. MHD mixed convection peristaltic flow with variable viscosity and thermal conductivity. Sains Malaysiana 43(10): 15831590.

He, J.H. 1999. Homotopy perturbation technique. Computer Methods in Applied Mechanics \& Engineering 178(3-4): 257-262.

Ijaz, N., Zeeshan, A., Bhatti, M.M. \& Ellahi, R. 2008. Analytical study on liquid-solid particles interaction in the presence of heat and mass transfer through a wavy channel. Journal of Molecular Liquids 250: 80-87.

Jaffrin, M.Y. 1973. Inertia and streamline curvature effects on peristalsis pumping. International Journal of Engineering Science 11(6): 681-699.

Jamil, D.F., Roslan, R., Abdulhameed, M. \& Hashim, I. 2018. Controlling the blood flow in the stenosed porous artery with magnetic field. Sains Malaysiana 47(10): 2581-2587.

Khan, M., Abbas, Q. \& Duru, K. 2010. Magnetohydrodynamic flow of a Sisko fluid in annular pipe: A numerical study. International Journal for Numerical Method in Fluids 62(10): $1169-1180$

Lauriola, I., Felisa, G., Petrolo, D., Di Federico, V. \& Longo, S. 2018. Porous gravity currents: Axisymmetric propagation in horizontally graded medium and a review of similarity solutions. Advances in Water Resources 115: 136-150.

Longo, S. \& Di Federico, V. 2015. Unsteady flow of shearthinning fluids in porous media with pressure-dependent properties. Transport in Porous Media 110(3): 429-447.

Mandal, P.K. 2005. An unsteady of non-Newtonian blood flow through tapered arteries with a stenosis. International Journal of Non-Linear Mechanics 40(1): 151-164.

Manton, M.J. 1975. Long-wavelength peristaltic pumping at low Reynolds number. Journal of Fluid Mechanics 68(3): 467-476.

Mehmood, O.U., Mustapha, N. \& Hayat, T. 2014. Partial slip effect on heat and mass transfer of MHD peristaltic transport in a porous medium. Sains Malaysiana 43(7): 1109-1118.

Mekheimer, K.S. \& El Kot, M.A. 2012. Mathematical modelling of unsteady flow os a Sisko fluid through an anisotropically tapered elastic arteries with time variant overlapping stenosis. Applied Mathematical Modelling 36(11): 53935407.

Sisko, A.W. 1958. The flow of lubricating greases. Industrial \& Engineering Chemistry 50(12): 1789-1792.

Nadeem, S. \& Akbar, N.S. 2009. Influence of heat transfer on a peristaltic transport of Herschel Bulkley fluid in a nonuniform inclined tube. Nonlinear Science \& Numerical Simulation 14(12): 4100-4113.

Nadeem, S. \& Akbar, N.S. 2010. Peristaltic flow of Sisko fluid in a uniform inclined tube. Acta Mechanica Sinica 26: 675-683.

Nichols, W.W. \& Orourke, M.F. 1973. McDonald's Blood Flow in Arteries. New York: Oxford University Press Inc.

Pal, D. \& Talukdar, B. 2011. Combined effects of Joul heating and chemical reaction on unsteady magnetohydrodynamic mixed convection of a viscous dissipating fluid over a vertical plate in porous media with thermal radiation. Mathematical and Computer Modelling 54(11-12): 30163036.

Rashid, M., Khan, M., Hayat, T., Imran Khan, M. \& Alsaedi, A. 2019. Entropy generation in flow of ferromagnetic liquid with nonlinear radiation and slip condition. Journal of Molecular Liquids 276: 441-452. 
Rohsenow, W.M., Hartnett, J.P. \& Cho, Y.I. 1998. Handbook of Heat Transfer. New York: McGraw-Hill.

Sadaf, H. \& Nadeem, S. 2017. Analysis of combined convective and viscous dissipation effects for peristaltic flow of Rabinowitsch fluid model. Journal of Bionic Engineering 14(1): 182-190.

Scheidegger, A.E. 1963. The Physics of Flow through Porous Media. New York: McGraw-Hill.

Shapiro, A.H., Jaffrin, M.Y. \& Weinberg, S.L. 1969. Peristaltic pumping with long wavelengths at low Reynolds number. Journal of Fluid Mechanics 37(4): 799-825.

Siddiqui, A.M., Azim, Q.A., Ashraf, A. \& Ghori, Q.K. 2008. Homotopy perturbation solution for peristaltic flow of a third order fluid. Topological Methods in Nonlinear Analysis 31: 331-339.

Sohail, M., Naz, R. \& Abdelsalam, S.I. 2019. On the onset of entropy generation for a nanofluid with thermal radiation and gyrotactic microorganisms through 3D flows. Physica Scripta. https://doi.org/10.1088/1402-4896/ab3c3f.
Verma, N. \& Parihar, R.S. 2010. Mathematical model of blood flow through a tapered artery with mild stenosis and hematocrit. Journal of Modern Mathematics and Statistics 4(1): 38- 43.

Department of Mathematics

Faculty of Education

Ain Shams University

Roxy, Cairo

Egypt

*Corresponding author; email: doaaroshdy@edu.asu.edu.eg

Received: 11 July 2019

Accepted: 15 January 2020 